\title{
Signal Transformation Approach to Tracking Control With Arbitrary References
}

\author{
Ali Bazaei, Member, IEEE, and S. O. Reza Moheimani, Fellow, IEEE
}

\begin{abstract}
In this paper, we introduce a signal transformation (ST) methodology for tracking control of a large class of arbitrary references. The method can improve tracking performance of ordinary one-degree-of-freedom (1-DoF) feedback control structure, while keeping robustness against unmodeled dynamics and limiting the projected measurement noise by ensuring a low closed-loop bandwidth. Using singular perturbation theory, sufficient conditions for stability and convergence of the tracking error are derived. Effectiveness of the proposed method is demonstrated by simulations. It is shown how ST method can provide a better control performance compared to ordinary 2-DoF feedback control systems having similar projected noise power, and maintain robustness against uncertainties, disturbances, and unmodeled dynamics.
\end{abstract}

Index Terms-Arbitrary reference tracking, hybrid control system, signal transformation, singular perturbation.

\section{INTRODUCTION}

$\mathbf{T}$ HERE are numerous applications in nanotechnology that require positioning accuracy of the order of a nanometer, or less [1], [2]. Such applications include scanning probe microscopy [3]-[5], nanometrology [6]-[9], nanolithography [10], [11], material science [12], [13], high-density data storage systems [14]-[16], and nano-fabrication [17]. Capacitive and inductive sensors are commonly used in nanopositioning systems due to their capability of providing simple solution for non-contact, high-resolution measurement. These sensors typically have a noise density of $20 \mathrm{pm} / \sqrt{\mathrm{Hz}}$ [18]. For every hundredfold increment in the closed-loop system bandwidth, the position accuracy of a nanopositioning scanner will decrease tenfold. This potentially degrades the resolution of the scanner, hindering it from performing positioning tasks that require subnanometer resolution. Hence, feedback control methods with limited closed-loop bandwidth are highly desirable in the presence of measurement noise.

Command pre-shaping methods can be considered as a possible way for vibration suppression in an already designed closed-loop control system, leaving the closed-loop bandwidth

Manuscript received October 13, 2010; revised May 14, 2011; accepted January 26, 2012. Date of publication February 03, 2012; date of current version August 24, 2012. This work was supported in part by the Australian Research Council Centre of Excellence for Complex Dynamic Systems and Control (CDSC), and in part by IBM Zurich Research Laboratories. Recommended by Associate Editor T. Zhou.

The authors are with the School of Electrical and Computer Engineering, The University of Newcastle Australia, Callaghan, NSW 2308, Australia, (e-mail: ali.bazaei@newcastle.edu.au; abazaei@yahoo.com; reza.moheimani@newcastle.edu.au).

Color versions of one or more of the figures in this paper are available online at http://ieeexplore.ieee.org.

Digital Object Identifier 10.1109/TAC.2012.2186474 of the measurement noise unaffected [19]-[28]. However, these methods are not suitable for tracking of time-varying commands such as triangular waveforms or suffer from lack of robustness to plant uncertainties. Some adaptive controllers are also used for tracking of time-varying [29]; however, they are prone to drift in integral adaptation gains or very high control demands in the presence of measurement noise [30]. Iterative learning control (ILC) can also be added as a feed-forward control action in a feedback system to improve the steady-state tracking error for repetitive references without altering the closed-loop bandwidth [31], [32]. However, it may require a large number of iterations to converge. Feedback control methods such as repetitive control (RC) for tracking of periodic references introduce large closed-loop bandwidths, which may not be acceptable in the presence of measurement noise. Moreover, the tradeoff between the tracking error and rejection of non-periodic disturbances in RC systems can cause problems when a multivariable plant has excessive cross coupling [33], [34].

In [35], the concept of signal transformation (ST) was put forward as a novel approach for tracking of triangular waveforms in a nanopositioning system. The method showed significant closed-loop performance improvement compared with an ordinary feedback control system having a similar control bandwidth [36], [37].

This paper describes how ST can be used along with traditional one-degree-of-freedom (1-DoF) feedback control methods to improve tracking error for a large class of arbitrary references. The method keeps the projected measurement noise below a pre-specified level to limit the adverse effects of sensor noise on the positioning accuracy of the closed-loop system. The remainder of the paper continues as follows.

\section{OBJECTIVES}

We first design appropriate ST mappings to cope with arbitrarily shaped reference signals. The resulting nonlinear switched-system is then analyzed using singular perturbation theory to obtain sufficient conditions for convergence of the tracking error to small values, where measurement noise and disturbances are ignored for simplicity. We present an example to show high control performance of ST method under a very low closed-loop bandwidth, along with numerical justification of the mathematical analysis and convergence criteria.

To deal with the problem of measurement noise in feedback control systems, concept of projected noise is introduced. As shown in Fig. 1, consider a typical feedback control system designed to control a physical quantity $x$, which is measured by a 


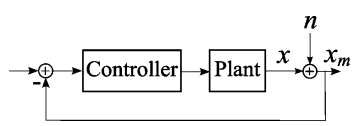

Fig. 1. Illustration of projected measurement noise in a typical feedback control system.

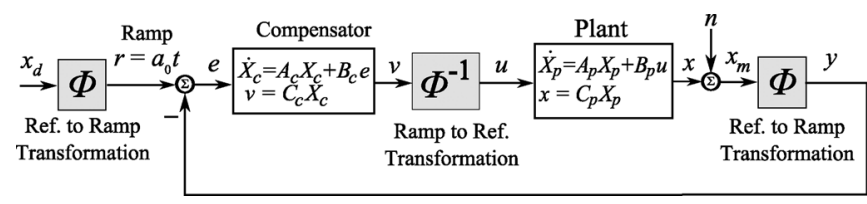

Fig. 2. Schematic diagram of signal transformation method.

sensor, that provides a measured signal $x_{m}:=x+n$ for feedback, which is affected by measurement noise $n$. By the projected noise, we mean the direct effect of the measurement noise signal $n$ on the actual controlled output $x$ in the closed-loop feedback system. For linear systems, this effect can be quantified in terms of the noise signal $n$ and the closed-loop transfer function from $n$ to $x$, which is denoted by $T_{x n}(s)$. An objective of this paper is to evaluate the control performance of ST method in tracking of arbitrary references while limiting the projected measurement noise power.

Finally, we consider a methodology to restore robustness against disturbances and uncertainties in plant DC gain for ST method, and the results are compared with ordinary 2-DoF feedback systems under equal measurement noise attenuations.

\section{Signal TRANSFORMATION}

ST approach, as proposed in [35], incorporates appropriate mappings between non-smooth signals (e.g., a triangular waveform as the reference) and smooth signals (e.g., a ramp) in a control system to improve the tracking error while keeping the closed-loop bandwidth low to limit the projected measurement noise. The ST method for control of a SISO plant is described by the hybrid control system shown in Fig. 2, where $\Phi$ and $\Phi^{-1}$ refer to the ST mappings. In this paper, the mapping $\Phi$ is designed to be capable of converting the reference signal to a ramp signal $r=a_{0} t$, where $a_{0}$ is the ramp slope. The ST block between the plant and compensator does the reverse action, i.e., it can convert the ramp signal into the reference signal. Consider an ideal situation, where the noise $n$ is zero, the plant is a unity gain transfer function, and its output is perfectly following the reference ramp signal. In this case, the input/output signals at compensator block will be smooth signals with no breaks or discontinuities and the burden of providing appropriate trajectories at the actuator, which demands a high control bandwidth in an ordinary feedback system, is carried by the ST block. In this way, the compensator can be designed with a smaller closed-loop bandwidth in favor of rejecting the projected measurement noise without deteriorating the steady-state error.

The ST methodology proposed in this paper is aimed at tracking a large class of time-varying reference signals. We assume that the reference signal $x_{d}(t)$ is continuous and periodic but may have a finite number of break points in each period $T$. Each period contains a number of strictly increasing and decreasing intervals (monotonic), within which the reference

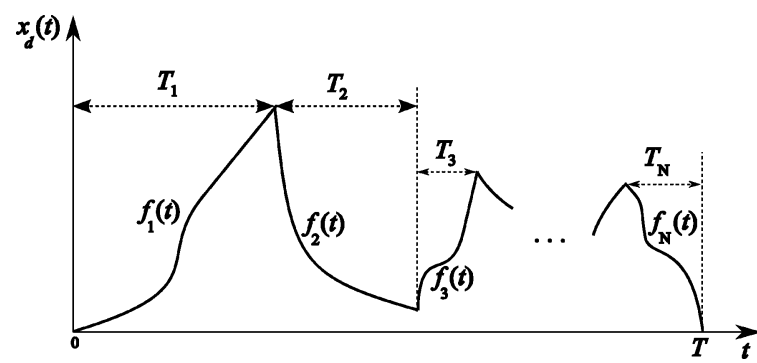

Fig. 3. Illustration of a continuous reference signal with period $T$.

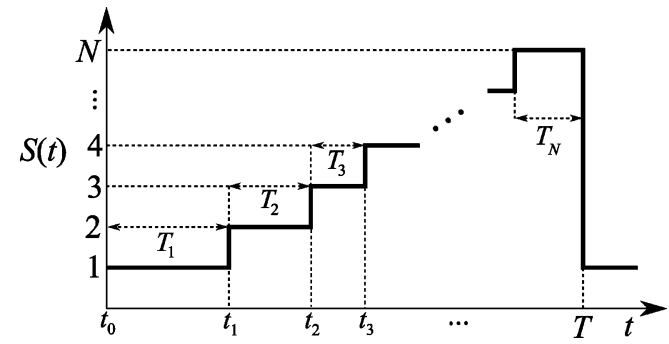

Fig. 4. Illustration of periodic signal $S(t)$ in a period.

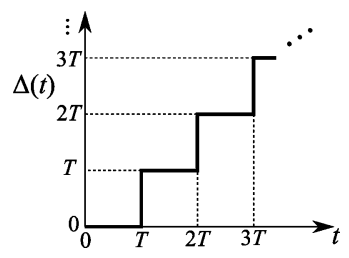

Fig. 5. Illustration of signal $\Delta(t)$.

signal has no break points and is sufficiently many times differentiable. A typical reference signal is shown Fig. 3, where $N$ is the number of the monotonic intervals in each period, $T_{i}(i=1, \ldots, N)$ refers to the interval of the $i$ th monotonic interval, and $f_{i}(t)$ describes the profile of the desired signal in the $i$ th monotonic interval of the first period. This description can be extended to non-periodic references if the period and/or the number of intervals tend to infinity. Discontinuities and constant intervals in the reference can also be approximated by lines with very steep and very small slopes, respectively. To design the ST mappings, we use a priori knowledge of the reference. Thus, functions $f_{1}(t), \ldots$, and $f_{N}(t)$, defined in Fig. 3, are available in advance.

\section{Design of TRANSFORMATION MAPPINGS}

The method that we propose to design the ST mappings incorporates the monotonic functions $f_{1}(t), \ldots$, and $f_{N}(t)$ and their inverses. The periodic reference signal $x_{d}(t)$ can be formulated in terms of the monotonic functions in the following form:

$$
x_{d}(t)=\sum_{i=1}^{N} \delta(S(t)-i) f_{i}(t-\Delta(t))
$$

where $S(t)$ and $\Delta(t)$ are piecewise constant signals described in Figs. 4 and 5. Function $\delta(\cdot)$ in (1) is the discrete impulse function defined as

$$
\delta(x):= \begin{cases}1, & \text { if } x=0 \\ 0, & \text { if } x \neq 0\end{cases}
$$


Signal $S(t)$ is a periodic signal equal to the index $i$ of the monotonic function being used to generate the reference signal in the corresponding time interval. Signal $\Delta(t)$ can be formulated in the following form:

$$
\Delta(t)=T \cdot \text { floor }\left(\frac{t}{T}\right)
$$

where function floor $(\cdot)$ refers to the largest integer not greater than its argument. Mapping $\Phi^{-1}$ in Fig. 2 is designed such that it can generate the reference signal at $u$ if input $v$ is driven by a ramp signal of $a_{0} t$. Using (1), we select the following relationship to realize mapping $\Phi^{-1}$ in Fig. 2:

$$
u=\Phi^{-1}(v, t)=\sum_{i=1}^{N} \delta(S(t)-i) f_{i}\left(\frac{v}{a_{0}}-\Delta(t)\right) .
$$

Using (4) and availability of the inverse functions $f_{1}^{-1}(t), \ldots$, and $f_{N}^{-1}(t)$, the mapping $\Phi$ in Fig. 2 is obtained in the following form:

$$
r=\Phi\left(x_{d}, t\right)=a_{0}\left[\Delta(t)+\sum_{i=1}^{N} \delta(S(t)-i) f_{i}^{-1}\left(x_{d}\right)\right] .
$$

Remark: The domain and range of function $f_{i}(\cdot)$ and its inverse are defined according to Fig. 3, which is all that is needed to convert the ramp signal to the reference signal and vice versa using (4) and (5), respectively. However, it is better to further extend the range and domain of each function in order to have well-defined output values for the ST blocks in the closed-loop system. Notice that the periodicity of the reference signal is not a required condition and has no effect on convergence of tracking error. The reasons for the periodicity assumption of the reference are that it allows a more convenient implementation and separation of transient and steady-state performances for the closed-loop system in a single run.

\section{Closed-Loop Stability}

In this section, we propose sufficient conditions for stability of the closed-loop system and convergence of the tracking error. We assume that the plant and compensator dynamics are of degrees $n_{p}$ and $n_{c}$ and are described by linear-time-invariant state-space matrix sets $\left[A_{p}, B_{p}, C_{p}\right]$ and $\left[A_{c}, B_{c}, C_{c}\right]$ with $X_{p}$ and $X_{c}$ referring to the corresponding state vectors, respectively, as shown in Fig. 2. The feedthrough matrices have been assumed zero. Assuming zero noise and defining $T_{0}:=0$, in the time interval of

$$
\Delta+\sum_{k=0}^{i-1} T_{k}<t<\Delta+\sum_{k=0}^{i} T_{k}
$$

signal $S$ is equal to $i$ and the following relationships hold:

$$
\begin{aligned}
u & =f_{i}\left(\frac{v}{a_{0}}-\Delta\right) \\
y & =a_{0}\left[\Delta+f_{i}^{-1}(x)\right] .
\end{aligned}
$$

In this way, the closed-loop dynamics in the time interval of (6) can be written by the following state-space equation:

$$
\dot{X}_{p}=A_{p} X_{p}+B_{p} f_{i}\left(a_{0}^{-1} C_{c} X_{c}-\Delta\right)
$$

$$
\dot{X}_{c}=A_{c} X_{c}-B_{c} a_{0} f_{i}^{-1}\left(C_{p} X_{p}\right)+B_{c} r_{e}(t)
$$

where $r_{e}:=r-a_{0} \Delta$ is a sawtooth signal with slope of $a_{0}$ and period of $T$. Equations (9) and (10) reveal that the closed-loop system dynamics are nonlinear in a monotonic time interval. Since the nonlinear functions change when one monotonic interval is switched to the next, the system has a time-varying nature as well. Exact analysis of nonlinear time-varying dynamic systems is generally a hard task. However, the analysis can be simplified under the following assumptions.

1) The plant has a stable transfer function with unity low frequency gain, i.e., $-C_{p} A_{p}^{-1} B_{p}=1$.

2) Each monotonic time interval of $T_{i}$, associated with the reference signal, is large compared to the open-loop settling time $t_{s}$ of the plant, for instance, $T_{i}>10 t_{s}$. This condition allows the tracking error to converge to low values in a small fraction of time interval of $T_{i}$.

3) The closed-loop system in Fig. 2 with ST blocks and plant replaced by unity gains is stable, has zero steady-state error for tracking a ramp reference, adequate stability margins (Phase Margin $>30^{\circ}$, Gain Margin $>10 \mathrm{~dB}$ ), and a bandwidth much less than the open-loop bandwidth of the plant. In other words, with ST blocks replaced by unity gains in Fig. 2, the state $X_{p}$ of the plant can change much faster than the state $X_{c}$ of compensator, during any transients caused by, for example, discontinuous disturbance signals.

Considering assumption 3, (9) can be regarded as a fast stable dynamics driven by a slow term of $B_{p} f_{i}\left(a_{0}^{-1} C_{c} X_{c}-\Delta\right)$ in the time interval of (6). Hence, after a short transient at the initial moments of the monotonic time interval of $T_{i}$, we can neglect $\dot{X}_{p}$ in (9) to obtain the following solution for the plant state vector in terms of the compensator state:

$$
X_{p} \simeq-A_{p}^{-1} B_{p} f_{i}\left(a_{0}^{-1} C_{c} X_{c}-\Delta\right)
$$

where existence of the inverse matrix is assured by assumption 1. Using (10), (11), and assumption 1, the following approximate closed-loop dynamics are obtained for the compensator state:

$$
\dot{X}_{c} \simeq\left(A_{c}-B_{c} C_{c}\right) X_{c}+B_{c} r .
$$

Equation (12) describes the closed-loop dynamics of the compensator, which is stable and much slower than that of the plant due to assumption 3 (the state matrix of $\left(A_{c}-B_{c} C_{c}\right)$ is the closed loop state matrix with ST blocks and plant replaced by unity gains in Fig. 2). According to assumption 3, the steady-state solution of LTI state-(12) to a ramp input provides a zero steady-state error. Hence, the compensator's input, which is equal to the tracking error of $e$ in Fig. 2, can converge to small values provided that the foregoing assumptions are satisfied.

\section{Singular Perturbation Analysis}

To cast the foregoing discussion into a more rigorous framework, singular perturbation analysis is used in this section for the proposed ST method. We assume that the plant is stable and 
has a unity DC gain as before with a state-space dynamic equation in the following form:

$$
\left\{\begin{array}{l}
\varepsilon \dot{X}_{p}=\widehat{A}_{p}(\varepsilon) X_{p}+\widehat{B}_{p}(\varepsilon) u \\
x=\widehat{C}_{p}(\varepsilon) X_{p}
\end{array}\right.
$$

where $\varepsilon$ is a positive small parameter, and matrices $\widehat{A}_{p}(\varepsilon)$, $\widehat{B}_{p}(\varepsilon)$, and $\widehat{C}_{p}(\varepsilon)$ are sufficiently many times continuously differentiable functions of $\varepsilon$. A sufficient condition for the existence of state-space representation (13) is that the poles and zeros of the plant are proportional to $1 / \varepsilon$, i.e., the plant transfer function approaches a unity gain as $\varepsilon$ tends to zero. This condition is justified in the Appendix, where we also offer a realization method to write state-space equations in the form of (13) for the plant. Using (7), (8), and considering the ramp signal $r=a_{0} t$ as an extra state, the closed-loop system over the time interval of (6) has the following autonomous state-space dynamics:

$$
\begin{aligned}
\dot{X}_{a} & =f\left(X_{a}, X_{p}, \varepsilon\right) \\
& =\left[\begin{array}{cc}
A_{c} X_{c}-B_{c} a_{0}\left\{f_{i}^{-1}\left[\widehat{C}_{p}(\varepsilon) X_{p}\right]+\Delta\right\}+B_{c} r \\
\dot{X}_{0}
\end{array}\right] \\
\varepsilon \dot{X}_{p} & =g\left(X_{a}, X_{p}, \varepsilon\right) \\
& =\widehat{A}_{p}(\varepsilon) X_{p}+\widehat{B}_{p}(\varepsilon) f_{i}\left(a_{0}^{-1} C_{c} X_{c}-\Delta\right)
\end{aligned}
$$

where $X_{a}:=\left[X_{c}^{T}, r\right]^{T}$ is an augmented state vector. We assume the monotonic functions $f_{i}(\cdot)$ and $f_{i}^{-1}(\cdot)$ are sufficiently many times continuously differentiable with respect to their arguments, and so are functions $f$ and $g$. Since the plant is stable, equality (15) at $\varepsilon=0$ has an isolated real root for $X_{p}$ as

$$
\bar{X}_{p}=-\bar{A}_{p}^{-1} \widehat{B}_{p} f_{i}\left(a_{0}^{-1} C_{c} \bar{X}_{c}-\Delta\right)
$$

where the bar over the parameters and state variables indicates that they belong to the system with $\varepsilon=0$, which is the slow subsystem. Hence, the closed-loop dynamics (14) and (15) conform with the "standard form" of singular perturbation [38]. Inserting for $X_{p}$ in (14) from (16) and using the unity DC gain of the plant while $\varepsilon$ is zero, the closed-loop "quasi-steady-state model" is obtained in the following form:

$$
\dot{\bar{X}}_{c}=\left(A_{c}-B_{c} C_{c}\right) \bar{X}_{c}+B_{c} r
$$

where the initial state at $t_{o}:=\Delta+\sum_{k=0}^{i-1} T_{k}$ is equal to that of the compensator. Considering $\tau:=\left(t-t_{o}\right) / \varepsilon$ as the stretched time variable during the transient and $\widehat{X}_{p}:=X_{p}-\bar{X}_{p}$ as the boundary layer correction, we obtain the following "boundary layer system" by freezing $\varepsilon=0$ and $X_{c}=\bar{X}_{c}=X_{c}\left(t_{o}\right)$ in (15) and (16):

$$
\frac{d \widehat{X}_{p}}{d \tau}=\widehat{A}_{p} \widehat{X}_{p}
$$

Using (16), the initial condition for the boundary layer system is obtained in the following form:

$$
\widehat{X}_{p}\left(t_{o}\right)=X_{p}\left(t_{o}\right)+\overline{\widehat{A}}_{p}^{-1} \widehat{\widehat{B}}_{p} f_{i}\left[a_{0}^{-1} C_{c} X_{c}\left(t_{o}\right)-\Delta\right] .
$$

Theorem VI.1: If $\widehat{A}_{p}$ is a Hurwitz matrix, then there exits a positive constant $\varepsilon^{*}$ such that for all $t \in\left[t_{o}, t_{o}+T_{i}\right]$ and $\varepsilon \in\left[0, \varepsilon^{*}\right]$ the following relationships hold uniformly 1 :

$$
\begin{aligned}
& X_{c}=\bar{X}_{c}+O(\varepsilon) \\
& X_{p}=\bar{X}_{p}+\widehat{X}_{p}+O(\varepsilon)
\end{aligned}
$$

where $\bar{X}_{c}$ and $\bar{X}_{p}$ satisfy (17) and (16) with initial condition $\bar{X}_{c}\left(t_{o}\right)=X_{c}\left(t_{o}\right)$, and $\widehat{X}_{p}$ satisfies (18) and (19). Moreover, given any time $t_{b} \in\left(t_{o}, t_{o}+T_{i}\right)$, there is a positive constant $\varepsilon_{b}<\varepsilon^{*}$ such that the following equality holds uniformly for $t \in\left[t_{b}, t_{o}+T_{i}\right]$ whenever $\varepsilon<\varepsilon_{b}$ :

$$
X_{p}=\bar{X}_{p}+O(\varepsilon) .
$$

Proof: From (15), $\partial g / \partial X_{p}$ at $\varepsilon=0$ is $\overline{\widehat{A}}_{p}$. Since $\overline{\widehat{A}}_{p}$ is a Hurwitz matrix, real parts of its eigenvalues are less than a fixed negative number. Hence, Assumptions 2.1, 3.1, and 3.2 in [38] are satisfied and the above assertions are valid according to Tikhonov's theorem (Theorems 3.1 and 9.1 in [38] and [39], respectively).

\section{ERROR PROPAGATION FOR COMPENSATOR}

The previous section provides approximations for the plant's and compensator's states in terms of quasi steady-state model's and boundary layer system's states, which are valid over one generic monotonic time interval. In this section, and the next one, we use the above results to obtain approximation of the tracking error, as the time increases such that the monotonic interval is switched to the next one.

In order to use approximations (20) and (21) over the next monotonic interval, state $\bar{X}_{c}$ should be re-initialized to the compensator's state at the starting moment of the new interval and so should $\widehat{X}_{p}(t)$ according to (19) with $\Delta^{+}+\sum_{k=0}^{i} T_{k}$ as $t_{o}{ }^{2}$ Because of the integral actions in the plant's and compensator's state-space dynamics and their zero feed-through terms, states $X_{c}(t)$ and $X_{p}(t)$ are continuous at the switching moment between every two adjacent monotonic intervals. For simplicity, we neglect the noise, assume $\Delta=0$, let $N$ tend to infinity, and define the $i$ th switching moment in the following form:

$$
t_{i}=\sum_{k=0}^{i} T_{k}, \quad i=0,1,2, \ldots
$$

Due to the third simplifying assumption in Section $\mathrm{V}$, the quasi steady-state model (17) has a steady-state solution of $X_{s s}(t)$ for state $\bar{X}_{c}$, which exactly satisfies the following equality:

$$
C_{c} X_{s s}(t)=r(t)=a_{0} t .
$$

Over the time interval of $t \in\left(t_{i-1}, t_{i}\right),(20)$ allows us to define an $O(\varepsilon)$ vector of $v_{i}(t, \varepsilon):=X_{c}-\bar{X}_{c}$, which means there exist

\footnotetext{
${ }^{1} \mathrm{~A}$ vector $v(t, \varepsilon) \in \Re^{n}$ is $O(\varepsilon)$ over an interval $\left[t_{1}, t_{2}\right]$ if there exist positive constants $\kappa$ and $\varepsilon_{0}$ such that $\|v(t, \varepsilon)\| \leq \kappa \varepsilon$ for all $\varepsilon \in\left[0, \varepsilon_{0}\right]$ and $t \in\left[t_{1}, t_{2}\right]$, where $\|\cdot\|$ is the Euclidean norm.

${ }^{2}$ The plus/minus sign in the superscripts of time variables indicates an infinitesimal amount of time after/before the underlaying variable.
} 
positive constants $\kappa_{i}$ and $\varepsilon_{i}$ such that the following relationship holds:

$$
\left\|v_{i}(t, \varepsilon)\right\|<\kappa_{i} \varepsilon, \quad \forall t \in\left(t_{i-1}, t_{i}\right) \text {, and } \forall \varepsilon \in\left[0, \varepsilon_{i}\right] .
$$

Since the quasi steady-state model is exponentially stable, there exist finite positive constants $\alpha$ and $\beta$ such that the following inequality is satisfied in the time interval of $t \in\left(t_{i-1}, t_{i}\right)$ :

$$
\left\|\bar{X}_{c}(t)-X_{s s}(t)\right\|<\beta\left\|X_{i-1}^{c}-X_{i-1}^{s s}\right\| e^{-\alpha\left(t-t_{i-1}\right)}
$$

where $X_{i}^{c}$ and $X_{i}^{s s}$ refer to $X_{c}(t)$ and $X_{s s}(t)$ at $t=t_{i}$, respectively. Using (20), (26), and (25) at $t=t_{i}^{-}$, and continuity of $X_{s s}$ and compensator state $X_{c}$, we obtain

$$
\begin{aligned}
\left\|X_{i}^{c}-X_{i}^{s s}\right\| & <\beta\left\|X_{i-1}^{c}-X_{i-1}^{s s}\right\| e^{-\alpha T_{i}}+\left\|v_{i}\left(t_{i}^{-}, \varepsilon\right)\right\| \\
& <\beta\left\|X_{i-1}^{c}-X_{i-1}^{s s}\right\| e^{-\alpha T_{i}}+\kappa_{i} \varepsilon
\end{aligned}
$$

where $\varepsilon \in\left[0, \min _{i} \varepsilon_{i}\right]$. Recursive use of inequality (27) yields the following upper bound on the compensator's state error at the $i$ th switching moment:

$$
\left\|X_{i}^{c}-X_{i}^{s s}\right\|<\left\|X_{0}^{c}-X_{0}^{s s}\right\| \beta^{i} e^{-\alpha t_{i}}+\gamma_{i} \varepsilon
$$

where

$$
\gamma_{i}:=\sum_{l=1}^{i} \kappa_{l} \beta^{i-l} e^{-\alpha\left(t_{i}-t_{l}\right)}, \quad i \geq 1
$$

and $\gamma_{0}$ is defined to be zero.

Theorem VII.1: With the increase of time, the compensator state error $X_{c}(t)-X_{s s}(t)$ converges to an $O(\varepsilon)$ vector if the following conditions are satisfied::

$$
\begin{aligned}
\lim _{t \rightarrow+\infty} \beta^{S(t)} e^{-\alpha t} & =0 \\
\gamma_{i} & <\gamma_{\max }, \quad \forall i \in\{1,2, \ldots\}
\end{aligned}
$$

where $\gamma_{\max }$ is a finite positive value.

Proof: Over the $i$ th monotonic interval $\left(t \in\left(t_{i-1}, t_{i}\right)\right)$, a similar reasoning that led to (27) yields

$$
\left\|X_{c}(t)-X_{s s}(t)\right\|<\beta\left\|X_{i-1}^{c}-X_{i-1}^{s s}\right\| e^{-\alpha\left(t-t_{i-1}\right)}+\kappa_{i} \varepsilon .
$$

Using (28) with $i$ replaced by $i-1$, we obtain the following upper bound for the norm of compensator's state error over the $i$ th monotonic interval:

$$
\begin{aligned}
\left\|X_{c}(t)-X_{s s}(t)\right\|<\left\|X_{0}^{c}-X_{0}^{s s}\right\| \beta^{i} e^{-\alpha t} & \\
& +\left[\kappa_{i}+\gamma_{i-1} \beta e^{-\alpha\left(t-t_{i-1}\right)}\right] \varepsilon .
\end{aligned}
$$

As time increases, condition (30) ensures that the first term in the right hand side of inequality (33) converges to zero. Since $t_{i-1}<t$ in the $i$ th monotonic interval, (31) ensures that the remaining term in the right-hand side of inequality (33) is less than $\left(\gamma_{\max } \beta+\kappa_{i}\right) \varepsilon$. In this way, we obtain

$$
\lim _{t \rightarrow+\infty}\left\|X_{c}(t)-X_{s s}(t)\right\|<\left(\gamma_{\max } \beta+\kappa_{\max }\right) \varepsilon
$$

where

$$
\kappa_{\max }:=\max _{i} \kappa_{i}
$$

Similarly, over the $i$ th monotonic interval, (28) and (26) lead to the following inequality:

$$
\begin{aligned}
\left\|\bar{X}_{c}(t)-X_{s s}(t)\right\|<\| X_{0}^{c}- & X_{0}^{s s} \| \beta^{i} e^{-\alpha t} \\
& +\left[\gamma_{i-1} \beta e^{-\alpha\left(t-t_{i-1}\right)}\right] \varepsilon .
\end{aligned}
$$

Using assumptions (30) and (31), (36) yields the following inequality, which establishes that the quasi-steady-state model's error of $\bar{X}_{c}(t)-X_{s s}(t)$ also converges to an $O(\varepsilon)$ vector:

$$
\lim _{t \rightarrow+\infty}\left\|\bar{X}_{c}(t)-X_{s s}(t)\right\|<\gamma_{\max } \beta \varepsilon .
$$

Using Corollary 9.1.7 in [40], (24), (36), and (37) yield the following relationship in the $i$ th monotonic interval:

$$
\begin{aligned}
& \left|C_{c} \bar{X}_{c}(t)-r(t)\right| \\
& \quad<\left\|C_{c}\right\|\left\{\left\|X_{0}^{c}-X_{0}^{s s}\right\| \beta^{i} e^{-\alpha t}+\left[\gamma_{i-1} \beta e^{-\alpha\left(t-t_{i-1}\right)}\right] \varepsilon\right\}
\end{aligned}
$$

which establishes:

$$
\lim _{t \rightarrow+\infty}\left|C_{c} \bar{X}_{c}(t)-r(t)\right| \leq\left\|C_{c}\right\| \gamma_{\max } \beta \varepsilon
$$

when condition (30) is satisfied.

\section{ERROR PROPAGATION FOR PlANT}

The reference-profile-function $f_{i}(\cdot)$ is a smooth function because it is assumed differentiable. Let $U_{i}$ and $U_{i}^{\prime}$ be finite upper bounds on magnitudes of $f_{i}(\cdot)$ and its derivative, respectively. Using the mean value theorem [41], we have the following inequalities in the $i$ th monotonic interval:

$$
\begin{gathered}
\left|f_{i}(t)-f_{i}\left(a_{0}^{-1} C_{c} \bar{X}_{c}\right)\right|<U_{i}^{\prime}\left|t-a_{0}^{-1} C_{c} \bar{X}_{c}\right| \\
\left|f_{i}(t)-f_{i}\left(a_{0}^{-1} C_{c} X_{c}\right)\right|<U_{i}^{\prime}\left|t-a_{0}^{-1} C_{c} X_{c}\right| .
\end{gathered}
$$

Over the time interval of (6), (1) reduces to $x_{d}=f_{i}(t)$. Using (21), (16), and the fact that $\widehat{C}_{p}(\varepsilon)$ is a well-defined function around $\varepsilon=0$, the tracking error of the plant output in the absence of noise can be expressed as

$$
\begin{aligned}
x_{d}-x=f_{i}(t)+\widehat{C}_{p}(\varepsilon) \overline{\widehat{A}}_{p}^{-1} \widehat{\widehat{B}}_{p} f_{i}\left(a_{0}^{-1} C_{c} \bar{X}_{c}\right) & \\
& -\widehat{C}_{p}(\varepsilon) \widehat{X}_{p}+O(\varepsilon) .
\end{aligned}
$$

Since the plant's DC gain is unity, coefficient $\widehat{C}_{p}(\varepsilon) \bar{A}_{p}^{-1} \widehat{\widehat{B}}_{p}$ in (42) can be uniformly approximated by its value at $\varepsilon=0$ and the following equality is valid:

$$
\widehat{C}_{p}(\varepsilon) \widehat{A}_{p}^{-1} \widehat{B}_{p}=-1+O(\varepsilon) .
$$


Using (42), (43), (24), (40), and (38), the following inequality is obtained in the $i$ th monotonic interval:

$$
\begin{aligned}
\left|x_{d}-x\right|<\frac{U_{i}^{\prime}}{a_{0}}\left\|C_{c}\right\| \| X_{0}^{c}-X_{0}^{s s} & \| \beta^{i} e^{-\alpha t} \\
& +\left|\widehat{C}_{p}(\varepsilon) \widehat{X}_{p}(t)\right|+O(\varepsilon)
\end{aligned}
$$

which leads to the following fact if condition (30) is satisfied.

$$
\lim _{t \rightarrow+\infty}\left|x_{d}(t)-x(t)\right| \leq\left|\widehat{C}_{p}(\varepsilon) \widehat{X}_{p}(t)\right|+O(\varepsilon) .
$$

Equations (44) and (45) reveal that in the $i$ th monotonic interval, when $t_{i-1}$ is sufficiently large to allow us to ignore the first transient term in the right-hand side of (44), the tracking error magnitude becomes less than $\left\|\widehat{C}_{p}\right\| \cdot\left\|\widehat{X}_{p}\right\|+O(\varepsilon)$. Since $\widehat{A}_{p}$ is a Hurwitz matrix and $\widehat{X}_{p}$ satisfies the boundary layer systems (18), there exist finite positive constants $\alpha^{\prime}$ and $\beta^{\prime}$ such that the following inequality holds:

$$
\left\|\widehat{X}_{p}(t)\right\|<\left\|\widehat{X}_{p}\left(t_{i-1}^{+}\right)\right\| \beta^{\prime} e^{-\left(\alpha^{\prime} / \varepsilon\right)\left(t-t_{i-1}\right)}, t \in\left(t_{i-1}, t_{i}\right) .
$$

The monotonic time interval $T_{i}$ is long compared to the settling time of the plant. Hence, (44)-(46) ensure that the tracking error converges to an $O(\varepsilon)$ value in an initial small fraction of $T_{i}$.

To examine the behavior of tracking error in the foregoing small fractions of time, we consider the evolution of vector of $X_{i}:=\widehat{X}_{p}\left(t_{i}^{+}\right)$as the switching index $i$ increases. From (21), we can define the following $O(\varepsilon)$ vector

$$
w_{i}(t, \varepsilon):=X_{p}(t)-\bar{X}_{p}(t)-\widehat{X}_{p}(t), t \in\left(t_{i-1}, t_{i}\right) .
$$

Hence, there exist positive constants $\mu_{i}$ and $\epsilon_{i}$ such that in the $i$ th monotonic interval we may write

$$
\left\|w_{i}(t, \varepsilon)\right\|<\mu_{i} \varepsilon, \quad \varepsilon \in\left[0, \epsilon_{i}\right] .
$$

Thanks to continuity of the plant state at the $i$ th switching moment, denoted by $X_{i}^{p}:=X_{p}\left(t_{i}\right)$, and using (16), we can eliminate $X_{i}^{p}$ between (47) at $t=t_{i}^{-}$and (19) at $t_{o}=t_{i}^{+}$to obtain the following equation:

$$
X_{i}=\overline{\widehat{A}}_{p}^{-1} \widehat{B}_{p} g_{i}(\varepsilon)+w_{i}\left(t_{i}^{-}, \varepsilon\right)+\widehat{X}_{p}\left(t_{i}^{-}\right)
$$

where

$$
g_{i}(\varepsilon):=f_{i+1}\left(a_{0}^{-1} C_{c} X_{i}^{c}\right)-f_{i}\left(a_{0}^{-1} C_{c} \bar{X}_{c}\left(t_{i}^{-}\right)\right) .
$$

Since matrix $\widehat{A}_{p}$ in the boundary layer system (18) is constant, the following closed form solution is available:

$$
\widehat{X}_{p}(t)=e^{(1 / \varepsilon) \widehat{\widehat{A}}_{p}\left(t-t_{i-1}\right)} X_{i-1}, t \in\left(t_{i-1}, t_{i}\right) .
$$

Hence, we can write a recursive expression in the following form:

$$
X_{i}=e^{(1 / \varepsilon) \widehat{\widehat{A}}_{p} T_{i}} X_{i-1}+\xi_{i}(\varepsilon), i \geq 1
$$

where

$$
\xi_{i}(\varepsilon):=\bar{A}_{p}^{-1} \widehat{B}_{p} g_{i}(\varepsilon)+w_{i}\left(t_{i}^{-}, \varepsilon\right), \quad i \geq 1 .
$$

The recursive (52), which is a time-varying discrete-time statespace equation, has the following closed-form solution:

$$
X_{i}=e^{(1 / \varepsilon) \widehat{\widehat{A}}_{p} t_{i}} X_{0}+\sum_{k=1}^{i} e^{(1 / \varepsilon) \widehat{\widehat{A}}_{p}\left(t_{i}-t_{k}\right)} \xi_{k}
$$

where $X_{0}=\widehat{X}_{p}\left(t_{0}^{+}\right)$. Using (40) at $t=t_{i}^{-}$, continuity of the reference $\left(f_{i}\left(t_{i}^{-}\right)=f_{i+1}\left(t_{i}^{+}\right)\right)$, and replacing $i$ by $i+1$ and $t=t_{i}^{+}$in (41), we obtain

$$
\left|g_{i}(\varepsilon)\right|<U_{i}^{\prime}\left|t_{i}-a_{0}^{-1} C_{c} \bar{X}_{c}\left(t_{i}^{-}\right)\right|+U_{i+1}^{\prime}\left|t_{i}-a_{0}^{-1} C_{c} X_{i}^{c}\right| .
$$

Using (55), (24), (33), (36), and (48), we obtain an upper bound for the norm of $\xi_{i}$ in the following form:

$$
\left\|\xi_{i}(\varepsilon)\right\|<\eta_{i} \beta^{i} e^{-\alpha t_{i}}+\rho_{i} \varepsilon
$$

where

$$
\begin{aligned}
\eta_{i}:= & \left\|X_{0}^{c}-X_{0}^{s s}\right\| \frac{\left\|C_{c}\right\|}{a_{0}}\left\|\widehat{\widehat{A}}_{p}^{-1} \widehat{\widehat{B}}_{p}\right\|\left(U_{i}^{\prime}+U_{i+1}^{\prime}\right) \\
\rho_{i}:= & \mu_{i}+\frac{\left\|C_{c}\right\|}{a_{0}}\left\|\widehat{\widehat{A}}_{p}^{-1} \widehat{B}_{p}\right\| \\
& \times\left[U_{i+1}^{\prime} \kappa_{i}+\left(U_{i}^{\prime}+U_{i+1}^{\prime}\right) \gamma_{i-1} \beta e^{-\alpha T_{i}}\right] .
\end{aligned}
$$

Since $\overline{\widehat{A}}_{p}$ is Hurwitz, $\left\|e^{-\widehat{\widehat{A}}_{p} \tau} V\right\|$ is less than $\|V\| \beta^{\prime} e^{-\alpha^{\prime} \tau}$ for any vector $V$ and positive scalar $\tau$. In this way, (56) and (54) lead to the following inequality:

$$
\left\|X_{i}\right\|<\left\|X_{0}\right\| \beta^{\prime} e^{-\left(\alpha^{\prime} / \varepsilon\right) t_{i}}+\delta_{i}+\sigma_{i} \varepsilon
$$

where

$$
\begin{aligned}
\delta_{i} & :=\beta^{\prime} \sum_{k=1}^{i} \eta_{k} \beta^{k} e^{-\left(\alpha^{\prime} / \varepsilon\right)\left(t_{i}-t_{k}\right)-\alpha t_{k}} \\
\sigma_{i} & :=\beta^{\prime} \sum_{k=1}^{i} \rho_{k} e^{-\left(\alpha^{\prime} / \varepsilon\right)\left(t_{i}-t_{k}\right)} .
\end{aligned}
$$

Assume the $\delta_{i}$ and $\sigma_{i}$ remain bounded with the increase of switching index $i$, with $\delta_{\infty}$ and $\sigma_{\infty}$ referring to their limit as $i$ tends to infinity, respectively. In this way (59) ensures that the $\left\|X_{i}\right\|-\delta_{\infty}$ remains below an $O(\varepsilon)$ value with the increase of switching index. Using (46) and (45), with the increase of time and in an $O(\varepsilon)$ sense, $\left\|\widehat{X}_{p}(t)\right\|$ and the tracking error converge to limits less than values proportional to $\delta_{\infty}$, which can be written as

$$
\begin{aligned}
\lim _{t \rightarrow+\infty}\left\|\widehat{X}_{p}(t)\right\| & \leq \beta^{\prime} \delta_{\infty}+O(\varepsilon) \\
\lim _{t \rightarrow+\infty}\left|x_{d}(t)-x(t)\right| & \leq\left\|\widehat{C}_{p}(\varepsilon)\right\| \beta^{\prime} \delta_{\infty}+O(\varepsilon) .
\end{aligned}
$$

Corollary VIII.1: Assume the measurement noise is zero and:

- The plant has a unity DC gain and its state-space dynamics depend on a small parameter $\varepsilon$, as described by (13), where $\widehat{A}_{p}(\varepsilon), \widehat{B}_{p}(\varepsilon)$, and $\widehat{C}_{p}(\varepsilon)$ have finite elements that are sufficiently many times differentiable with respect to $\varepsilon$ in an interval of $\varepsilon \in\left[0, \varepsilon^{*}\right]$

- Matrix $\left.\widehat{A}_{p}(\varepsilon)\right|_{\varepsilon=0}$ is a Hurwitz matrix.

- The state-space dynamics of compensator has no feedthrough matrix and provides stability and zero 
steady-state error for the ordinary feedback system with ramp reference, when the plant is replaced by a unity gain.

- Parameter $\varepsilon$ is small enough for (20) and (21) to hold.

- The reference signal is finite and continuous. In each monotonic interval, it is smooth with bounded derivative. Moreover, the monotonic intervals are long enough for conditions (30), (31), $\sigma_{i}<\infty$, and $\delta_{i}<\infty$ to hold,

then the steady-state absolute error in the feedback system with $S T$ converges, in an $O(\varepsilon)$ sense, to values not greater than $\left\|\widehat{C}_{p}(\varepsilon)\right\| \beta^{\prime} \delta_{\infty}$.

The assumptions on boundedness of $\delta_{i}$ and $\sigma_{i}$ are not very restrictive. For example when the duration of each monotonic interval has positive and limited lower and upper bounds $T_{\min }$ and $T_{\max }$, respectively, we can obtain limited upper bounds for $\delta_{\infty}$ and $\sigma_{\infty}$. Due to condition (31), there are finite upper bounds on $\eta_{i}$ and $\rho_{i}$, denoted by $\eta_{\max }$ and $\rho_{\max }$, respectively. After some manipulations, we obtain the following upper bounds for $\delta_{i}$ and $\sigma_{i}$ :

$$
\begin{aligned}
\delta_{i} & \leq \beta^{\prime} \eta_{\max } \frac{\beta e^{-\left(\alpha+\left(\alpha^{\prime} / \varepsilon\right) i\right) T_{\min }}-\left(\beta e^{-\alpha T_{\min }}\right)^{i+1}}{e^{-\left(\alpha^{\prime} / \varepsilon\right) T_{\min }}-\beta e^{-\alpha T_{\min }}} \\
\sigma_{i} & \leq \beta^{\prime} \rho_{\max } \frac{1-e^{-\left(\alpha^{\prime} / \varepsilon\right) T_{\min } i}}{1-e^{-\left(\alpha^{\prime} / \varepsilon\right) T_{\min }}}
\end{aligned}
$$

where the inequality $t_{i}-t_{k} \geq(i-k) T_{\min }$ for $i \geq k$ and $t_{k} \geq$ $k T_{\min }$ have been used. Similarly, the following inequalities also hold:

$$
\begin{aligned}
\beta^{S(t)} e^{-\alpha t} & \leq \beta \lambda^{i-1}, \quad t \in\left(t_{i-1}, t_{i}\right) \\
\gamma_{i} & \leq \kappa_{\max } \frac{1-\lambda^{i}}{1-\lambda}
\end{aligned}
$$

where $\lambda:=\beta e^{-\alpha T_{\min }}$. Using inequalities (66) and (67), a sufficient condition for simultaneous satisfaction of conditions (30) and (31) is as

$$
\lambda<1
$$

with $\gamma_{\max }=\kappa_{\max } /(1-\lambda)$. Assuming condition (68) is satisfied, inequality (65) shows that $\sigma_{i}$ and $\sigma_{\infty}$ are upper bounded by $\beta^{\prime} \rho_{\max } /\left(1-\exp \left(-\alpha^{\prime} T_{\min } / \varepsilon\right)\right)$. In this way, inequalities (64) and (68) reveal that as the switching index $i$ tends to infinity, the upper bound on $\delta_{i}$ vanishes and we have

$$
\delta_{\infty}=0 .
$$

Hence, when the other conditions in corollary VIII.1 are also met, only the $O(\varepsilon)$ terms in the right-hand sides of (62) and (63) remain. Thus, the boundary layer state $\widehat{X}_{p}$ as well as the steady-state error converge to zero in the $O(\varepsilon)$ sense.

Corollary VIII.2: If the assumptions in Corollary VIII.1 are met, then a sufficient condition for convergence of the tracking error $x_{d}-x$ and the boundary layer state $\widehat{X}_{p}$ to $O(\varepsilon)$ values is that parameter $\lambda:=\beta e^{-\alpha T_{\min }}$ is less than unity, where $T_{\min }$ is the least duration of the monotonic intervals of the reference signal and $\alpha$ and $\beta$ are positive constants only depending on compensator parameters as defined just before (26).

Remark: Notice that the results in Corollary VIII.2 still hold even if the reference signal has break points at the switching moments between the successive monotonic intervals. In contrast, for an ordinary feedback system with fixed compensator dynamics, such break points in the reference do not let the tracking
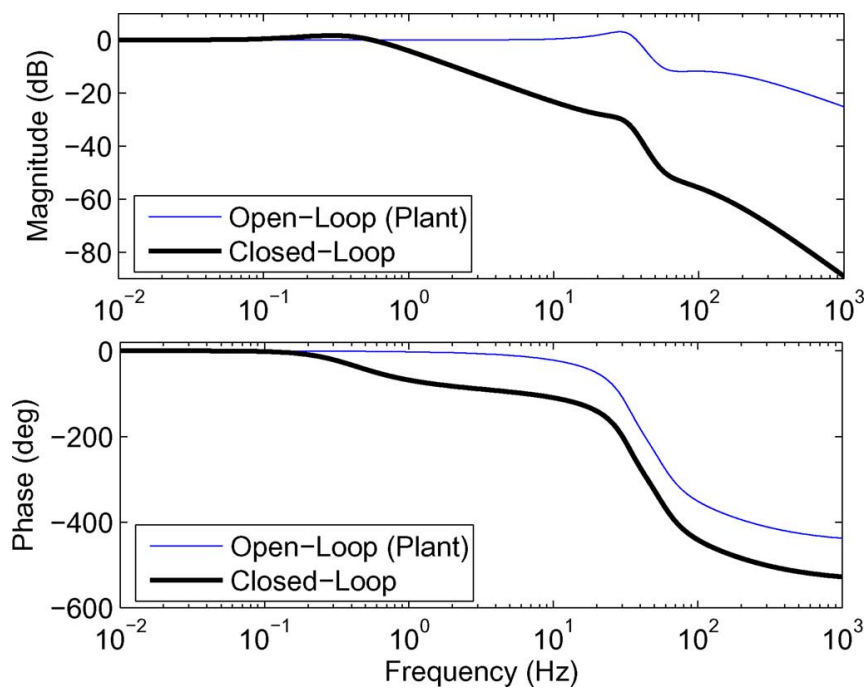

Fig. 6. Frequency responses of open-loop plant and closed-loop system with ST blocks replaced by unity gains.

error converge to arbitrarily small values, irrespective of the speed of the plant open-loop dynamics.

\section{ILLUSTRATIVE EXAMPLE}

For simulation purposes, the plant was considered as a unity-DC-gain non-minimum-phase transfer function with poles and zeros located at $\{-60 \pm 200 i,-1000\}$ and $\{120 \pm 330 i\} \mathrm{rad} / \mathrm{s}$, respectively. The compensator was selected as a linear combination of an integrator and a double integrator, described by $(4 s+6) / s^{2}$. Compared to $42 \mathrm{~Hz}$ open- loop bandwidth and $0.085 \mathrm{~s}$ settling time of the plant, the closed- loop system with ST blocks replaced by unity gains, has a low bandwidth of $0.88 \mathrm{~Hz}$, settling time of $2.3 \mathrm{~s}$, and dominant poles located at $-2.03 \pm 1.42 i \mathrm{rad} / \mathrm{s}$. Such reduction of closed-loop bandwidth is generally done to obtain robustness against unmodeled high frequency modes and reduction of projected measurement noise. Fig. 6 shows the bode diagram of the plant and the closed-loop system with signal-transformation blocks replaced by unity gains. The following functions were arbitrarily selected to construct the first period of the reference signal:

$$
\begin{aligned}
& f_{1}(t)=1.0373 \tanh (2 t-2), t \in[0,2) s \\
& f_{2}(t)=0.2035 \tan [0.9133(3.5-t)], t \in[2,5) s \\
& f_{3}(t)=2 t-11, t \in[5,6.5) s \\
& f_{4}(t)=2-3.056\left(1-e^{26-4 t}\right), \quad t \in[6.5,7.5) s .
\end{aligned}
$$

To implement the ST block $\Phi$ acting on the plant output $((8))$, functions $\tanh ^{-1}(\cdot)$ and $\ln (\cdot)$ were forced to generate limited and real values, by limiting their arguments within ranges $[-0.99,+0.99]$ and $\left[3.554 \times 10^{-4},+\infty\right)$, respectively. Moreover, the argument of tangent function in block $\Phi^{-1}((4))$ was restricted within the range of $[-1.5,1.5]$ rad to prevent unlimited values at $\pm \pi / 2$.

With a unity ramp slope and zero initial conditions, the closed- loop response of plant output with ST along with the reference signal are shown in Fig. 7. Clearly, the plant output closely follows the reference. If we replace the ST blocks 


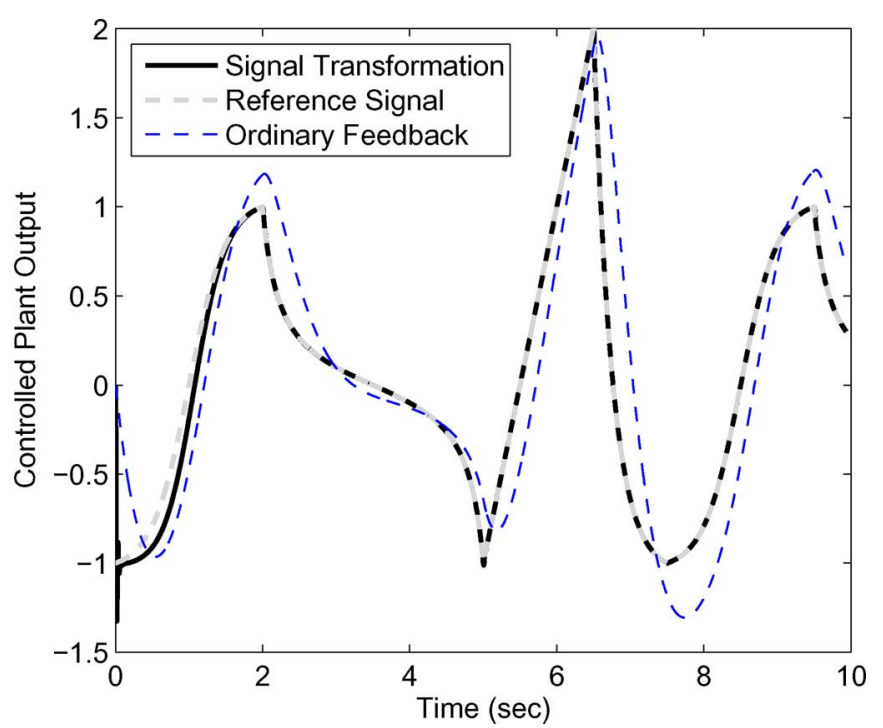

Fig. 7. Controlled plant output with the proposed ST method along with the reference signal. The controlled plant output with ordinary feedback structure is also included.

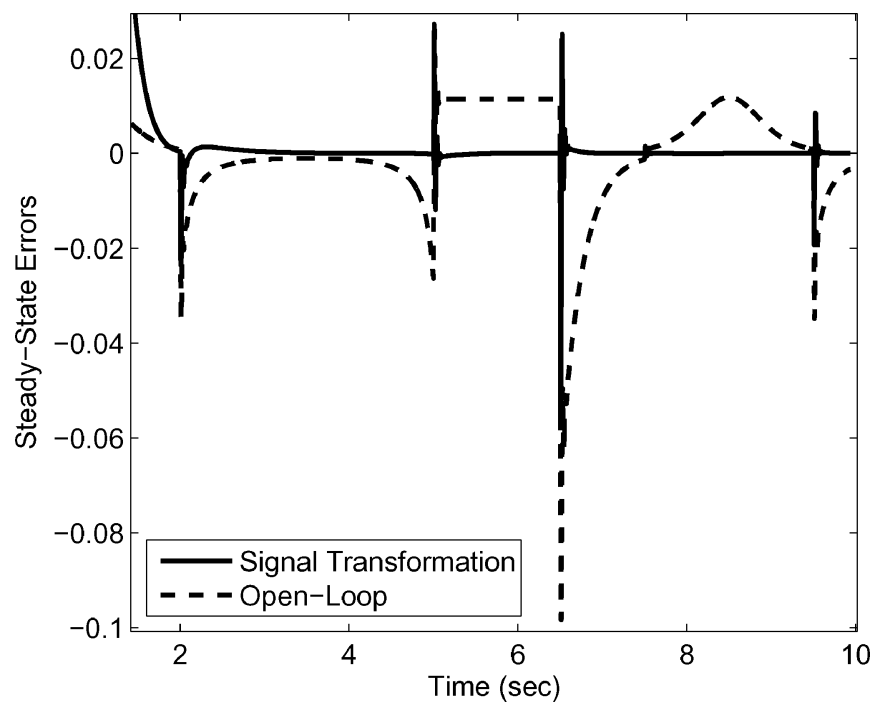

Fig. 8. Comparison of the steady-state tracking errors obtained by ST and open-loop methods.

by unity gains, the controlled output, also included in Fig. 7, cannot acceptably follow the reference. Thus, with ST, we can keep the closed-loop bandwidth as low as the ordinary system $(0.88 \mathrm{~Hz})$, while considerably improving the tracking performance in comparison with the ordinary system. Fig. 8 shows the tracking error with ST along with the tracking error associated with open-loop application of the reference at the plant input. Although the open-loop method uses a high bandwidth of $42 \mathrm{~Hz}$ (at the expense of no robust performance against unmodeled high-frequency dynamics), its steady-state tracking performance is worse than the ST method, which uses the low bandwidth of $0.88 \mathrm{~Hz}$.

Let us justify the stability criteria in Section $\mathrm{V}$ through this example. Selecting $\varepsilon=1 / 30$ as the ratio of the real part of the dominant poles of plant to that of the quasi-steady-state model and using the procedure in the Appendix, the plant's state-space realization (13) is obtained as

$$
\begin{aligned}
\widehat{A}_{p} & =\left[\begin{array}{ccc}
-33 . \overline{3} & 1 & 0 \\
0 & -2 & 6 . \overline{6} \\
0 & -6 . \overline{6} & -2
\end{array}\right], \widehat{B}_{p}=\left[\begin{array}{l}
0 \\
0 \\
1
\end{array}\right] \\
\widehat{C}_{p}^{T} & =\left[\begin{array}{c}
2678.2 \\
-76.6 \\
11.8
\end{array}\right] .
\end{aligned}
$$

The state space matrices associated with the compensator can be written as

$$
A_{c}=\left[\begin{array}{ll}
0 & 0 \\
1 & 0
\end{array}\right], B_{c}=\left[\begin{array}{l}
0 \\
1
\end{array}\right], C_{c}^{T}=\left[\begin{array}{c}
1 \\
1.5
\end{array}\right]
$$

To determine values for $\alpha$ and $\beta$ defined in (26), we need to determine an upper bound on Euclidean norm of vector $W:=$ $e^{\left(A_{c}-B_{c} C_{c}\right) t} V$ in terms of $\|V\|$. Assuming $V=\left[v_{1}, v_{2}\right]^{T}$, vector $W$ has the following expression in this example:

$$
\begin{aligned}
& W=e^{-2 t} \\
& \times\left[\begin{array}{c}
\cos (\sqrt{2} t)-\sqrt{2} \sin (\sqrt{2} t)] v_{1}-3 \sqrt{2} \sin (\sqrt{2} t) v_{2} \\
\frac{\sqrt{2}}{2} \sin (\sqrt{2} t) v_{1}+[\cos (\sqrt{2} t)+\sqrt{2} \sin (\sqrt{2} t)] v_{2}
\end{array}\right] .
\end{aligned}
$$

Since the Euclidean norm of a vector cannot exceed the sum of absolute values of its elements, the following inequalities are obtained after some manipulations:

$$
\begin{aligned}
\left\|e^{\left(A_{c}-B_{c} C_{c}\right) t} V\right\| & \leq e^{-2 t}\left[\left(\sqrt{3}+\frac{\sqrt{2}}{2}\right)\left|v_{1}\right|+(3 \sqrt{2}+\sqrt{3})\left|v_{2}\right|\right] \\
& \leq 6.4534 e^{-2 t}\|V\| .
\end{aligned}
$$

In this way, we can assign the following values to $\alpha$ and $\beta$.

$$
\alpha=2, \quad \beta=6.4534 \text {. }
$$

In this example we have $T_{\min }=1 \mathrm{~s}$. Hence, parameter $\lambda$ is obtained as

$$
\lambda:=\beta e^{-\alpha T_{\min }}=0.8734<1
$$

which reveals that condition (68) is satisfied. As mentioned in the Remark, satisfaction of condition (68) ensures that conditions (30) and (31) are also satisfied. With the selected $\widehat{A}_{p}$ in (73) and after some manipulation ${ }^{3}$, we can also assign

${ }^{3}$ For an arbitrary three element vector $V=\left[v_{1}, v_{2}, v_{3}\right]^{T}$ we have

$$
\begin{aligned}
W:=e^{\widehat{\widehat{A}}_{p} t} V= & {\left[\begin{array}{c}
{\left[e^{-2 t}(a \cos \phi+b \sin \phi)-a e^{-33 . \overline{3} t}\right] v_{2}} \\
0 \\
0
\end{array}\right] } \\
& +\left[\begin{array}{c}
e^{-33 . \overline{3} t} v_{1}+\left[e^{-2 t}(a \sin \phi-b \cos \phi)+b e^{-33 . \overline{3} t}\right] v_{3} \\
e^{-2 t}\left(v_{2} \cos \phi+v_{3} \sin \phi\right) \\
e^{-2 t}\left(v_{3} \cos \phi-v_{2} \sin \phi\right)
\end{array}\right]
\end{aligned}
$$

where $a=141 / 4618, b=15 / 2309$, and $\phi:=6 . \overline{6} t$. Using the facts $\|W\| \leq$ $\left|w_{1}\right|+\sqrt{w_{2}^{2}+w_{3}^{2}}$ and $\left|v_{2}\right|+\left|v_{3}\right| \leq \sqrt{2\left(v_{2}^{2}+v_{3}^{2}\right)}$, we have

$$
\begin{aligned}
\left\|e^{\overline{\widehat{A}}_{p} t} V\right\| & \leq e^{-33 . \overline{3} t}\left|v_{1}\right|+\sqrt{v_{2}^{2}+v_{3}^{2}}\left(\sqrt{2} a e^{-33 . \overline{3} t}+c e^{-2 t}\right) \\
& \leq\left(\sqrt{1+2 a^{2}} e^{-33 . \overline{3} t}+c e^{-2 t}\right) \sqrt{v_{1}^{2}+v_{2}^{2}+v_{3}^{2}} \\
& \leq\left(c+\sqrt{1+2 a^{2}}\right) e^{-2 t}\|V\|
\end{aligned}
$$

where $c=1+\sqrt{2\left(a^{2}+b^{2}\right)}=1.0441$. Hence, we can select $\alpha^{\prime}=2$ and $\beta^{\prime}=c+\sqrt{1+2 a^{2}}$ in (46). 


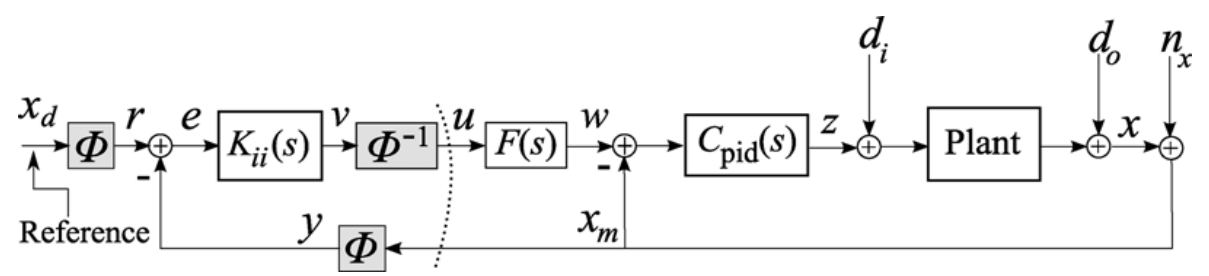

Fig. 9. Schematic diagram of robustified signal transformation method.

the following values to parameters $\alpha^{\prime}$ and $\beta^{\prime}$ defined before inequality (46):

$$
\alpha^{\prime}=2, \quad \beta^{\prime}=2.0451
$$

\section{ROBUSTNESS}

The foregoing example exhibited how ST can improve the tracking error in a low bandwidth feedback system. As the design method does not rely on the plant knowledge (except for its unity DC gain), it can have robustness with respect to variations in poles, zeros, and unmodeled dynamics. However, when the ST method is designed as described in Fig. 2, it becomes sensitive to input/output disturbances and variations in plant DC gain. A common way for providing robust unity DC gain and rejecting constant disturbances is to wrap an appropriate loop around the original plant with an integral action controller. The foregoing loop, which has a robust unity DC gain, can then be regarded as the plant block in the original ST structure in Fig. 2, which can be called robustified signal transformation (robustified ST) method. Effectiveness of this method for triangular references has been investigated in [36], [37].

In this section, we investigate the performance of the robustified ST method for tracking of arbitrary references, in the presence of measurement noise, constant disturbances, and variations in plant DC. The results are also compared with the ordinary two-degree-of-freedom (2-DoF) feedback control system having the same noise rejection performance.

Fig. 9 shows the components of the robustified ST method. The nominal plant, whose frequency response is shown in Fig. 10(a) and (c), has a unity DC gain, a zero at $-200 \mathrm{rad} / \mathrm{s}$, and lightly damped poles of $-1 \pm 99.995 i \mathrm{rad} / \mathrm{s}$. Due to disturbances, parameter variations, and highly vibrating behavior of the plant, open-loop controllers are not applicable. Assuming that the outer loop in Fig. 9 is temporarily open, the following PID controller in the inner loop:

$$
C_{\text {pid }}=4.544+\frac{627.2}{s}+\frac{0.0422 s}{1+0.005 s}
$$

provides an unlimited gain margin, a phase margin of $79.8^{\circ}$, desirable stable poles at $-503,-200$, and $-73.9 \pm 83.7 \mathrm{rad} / \mathrm{s}$, and a bandwidth of $121.6 \mathrm{~Hz}$ for the transfer function $T_{x w}(s)$ from $w$ to $x$ in Fig. 9, whose bode plot is also shown in Fig. 10(a) and (c) with dotted lines. For the robustified ST method in Fig. 9, the filter $F(s)$ was selected as

$$
F(s)=\frac{1+\frac{s}{764}}{1+\frac{s}{9932}}
$$
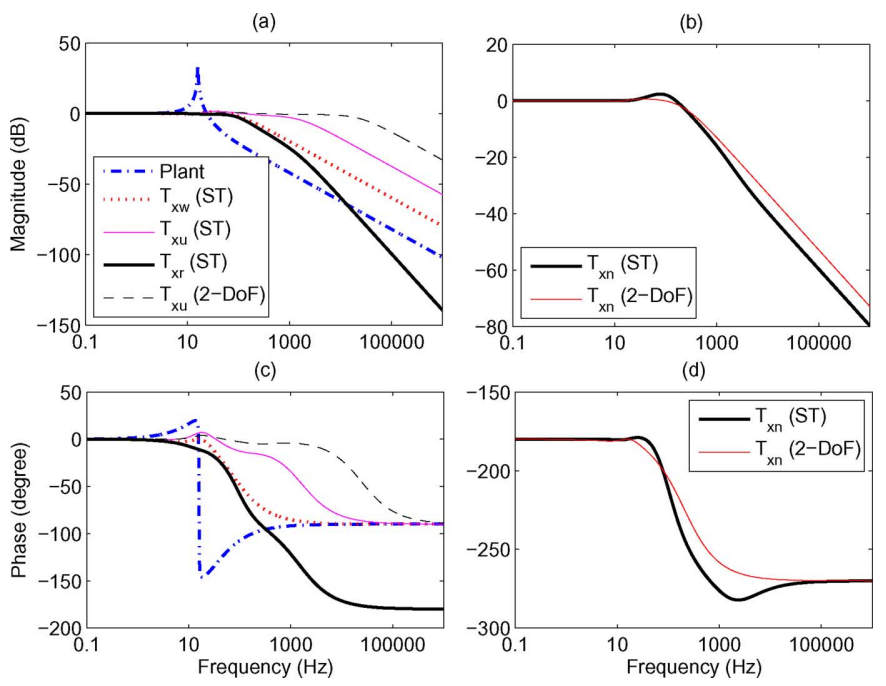

Fig. 10. Frequency responses.

to provide a higher bandwidth of $1060 \mathrm{~Hz}$ for the transfer function $T_{x u}(s)$, which is shown in Fig. 10(a) and (c) with thin solid curves, and can be regarded as the equivalent plant in Fig. 2. The compensator $K_{i i}(s)$ has a double integral action described as

$$
K_{i i}(s)=\frac{500+2000 s}{s^{2}} .
$$

With the ST blocks replaced by unity gains, the outer loop has an infinite gain margin, a phase margin of $78^{\circ}$, and a bandwidth of $103 \mathrm{~Hz}$ for closed-loop forward transfer function $T_{x r}(s)$, whose bode plot is shown in Fig. 10(a) and (c) with thick solid curves. In this case, the noise feedback bandwidth, which is defined as the bandwidth of the transfer function $T_{x n}(s)$ (also shown in Fig. 10(b) and (d) with thick solid curves), is $236.4 \mathrm{~Hz}$. Having the elements of the inner loop fixed in this example, we cannot considerably reduce the noise feedback bandwidth, as before, by lowering the gains of double integral compensator. Moreover, if filter $F(s)$ is replaced by a constant unity gain, the noise feedback bandwidth is just reduced to $182 \mathrm{~Hz}$. Hence, the high frequency amplification of filter $F(s)$ has a minor effect on increase of noise feedback bandwidth while it significantly expedites the initial decay of transient responses, as will be described in Fig. 17(a) and (b).

For comparison purposes, an ordinary two-degree-offreedom (2-DoF) feedback system is also considered. The 2-DoF structure is similar to the right-hand side of the dotted line in Fig. 9, where the reference signal $x_{d}$ is directly fed into input $u$ of the prefilter $F(s)$ without using any signal transformation. The gains of the PID controller (80) for the 
(a)

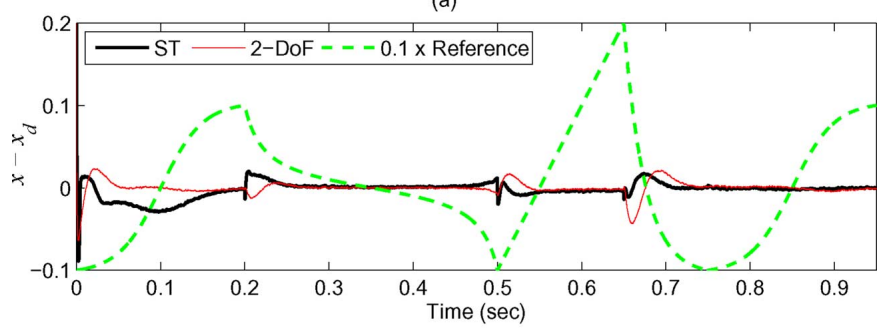

(b)

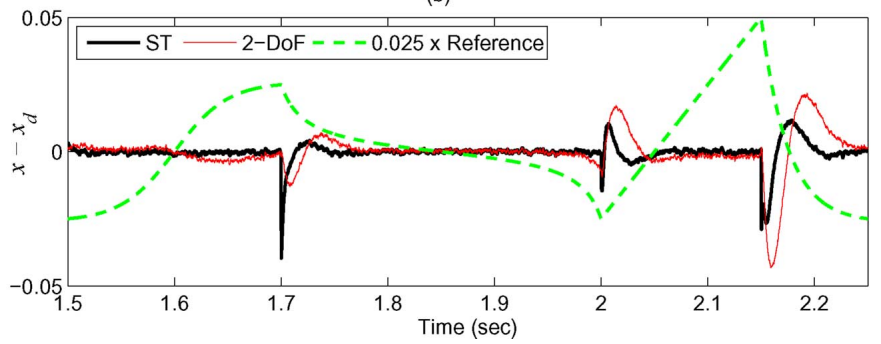

Fig. 11. Tracking errors with robustified signal transformation (ST) and ordinary 2-DoF methods with measurement noise. (a) Transients. (b) Steady-state.
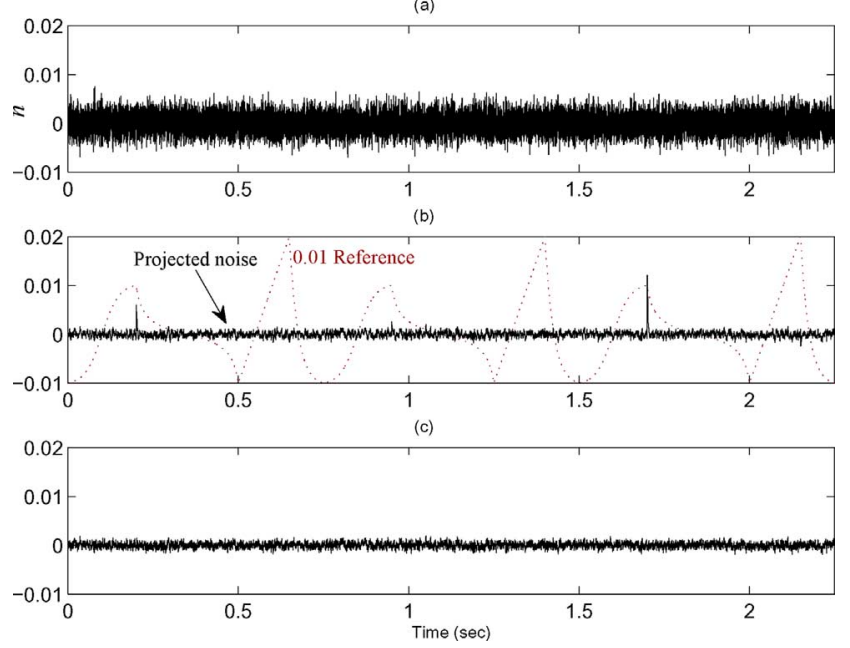

Fig. 12. Time histories of measurement noise (a), and projected noises in robustified ST (b) and in the ordinary 2-DoF (c) systems. (a) Measurement noise source. (b) Projected noise in ST system. (c) Projected noise in 2-DoF system.

2-DoF system were increased by a factor of 2.1875 such that the projected noise has almost the same standard deviation as that of the ST method. The closed-loop noise transfer function $T_{x n}(s)$ is shown in Fig. 10(b) and (d) with thin solid curves and has a bandwidth of $244 \mathrm{~Hz}$. The following prefilter $F(s)$ was tuned to provide a bandwidth of $20 \mathrm{kHz}$ for the forward transfer function $T_{x u}(s)$, which is also shown in Fig. 10(a) and (c) with dashed lines:

$$
F(s)=\frac{1+\frac{s}{1533}}{1+\frac{s}{153300}} .
$$

Fig. 11 shows the tracking errors of the above ST and 2-DoF methods, affected by a common measurement noise signal, whose waveform is shown in Fig. 12(a). The reference signals for both systems are similar to that of the previous example but ten times faster. Fig. 13 shows clearer views of the steady-state
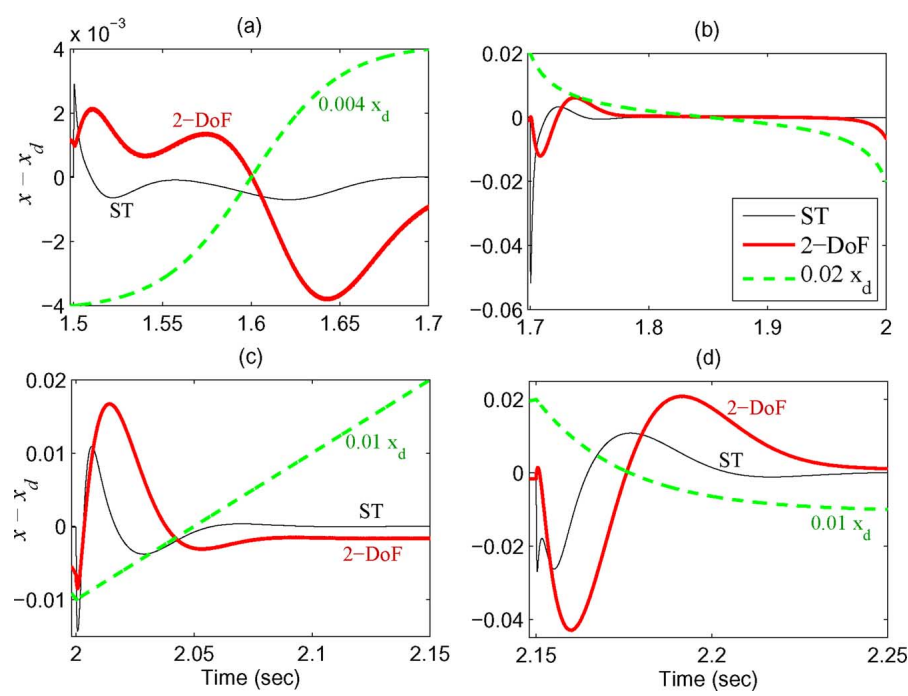

Fig. 13. Close-ups of steady-state tracking errors in the four monotonic time intervals of the reference in the absence of noise for robustified ST and ordinary 2-DoF systems, having the same projected noise powers.

error during the four monotonic intervals of the reference in the absence of noise, where root-mean-square (RMS) values of the steady-state errors in ST and 2-DoF systems are 0.0042 and 0.0073 , respectively. Clearly, with the ST method the resulting steady-state performance is much better than that of the ordinary 2-DoF system. To investigate the effect of the nonlinear mappings on the projected noise, a counterpart of the ST system was concurrently run without any noise source. Fig. 12(b) shows the difference between the plant outputs in the two ST methods, as an indication of the projected noise in ST system. A similar procedure was simultaneously performed for the 2-DoF system, and the corresponding projected noise is shown in Fig. 12(c). Mostly, the projected noise in the ST system is similar to that of the 2-DoF system. Hence, the nonlinear mappings in the ST method have a very minor effect on the projected noise. This fact may be justified by the inverse operations of the two nonlinear blocks $\Phi$ and $\Phi^{-1}$ in the outer loop in Fig. 9. The projected noise in the ST system has minor spikes that appear at some of the switching moments of the reference, which is due to discontinuity of signals $S(t)$ and $\Delta(t)$ in the nonlinear mappings.

In order to investigate robustness of the two control methods against uncertainty in plant DC gain, its value was changed five times more and less than the nominal unity value and the resulting steady-state errors are shown in Figs. 14 and 15, respectively, where the noise was eliminated for better clarity. In Fig. 14, where plant DC gains is 5, the RMS values of the steadystate errors for ST and 2-DoF methods are 0.0064 and 0.0111 , respectively; and in Fig. 15, where the DC gain is 0.2, the RMS values increase to 0.0241 and 0.048 , respectively. Clearly, the steady-state performance of the ST method is acceptable and better than that of the ordinary 2-DoF method, even after unknown and remarkable changes in plant DC gain values. In contrast to the 2-DoF method, the ST method progressively improves the steady-state error in each monotonic interval, regardless of how hard it is to follow the reference [e.g., see Fig. 14(b)]. 

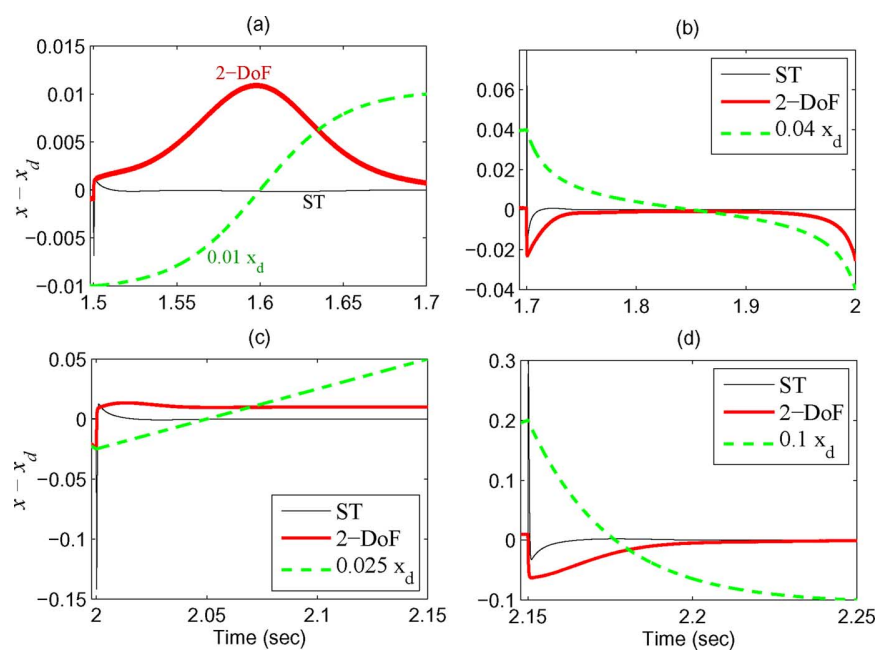

Fig. 14. Close-ups of steady-state tracking errors in the absence of noise for robustified ST and ordinary $2-\mathrm{DoF}$ systems, after a fivefold increase in plant DC gain. (a)

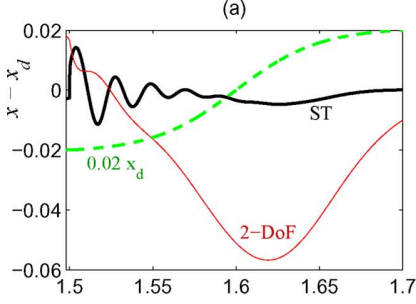

(c)

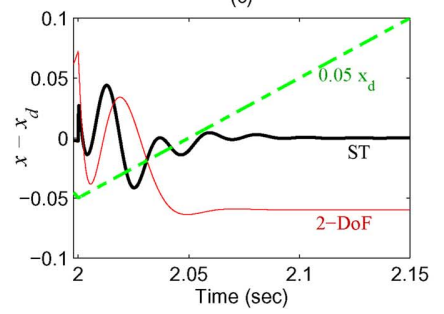

(b)

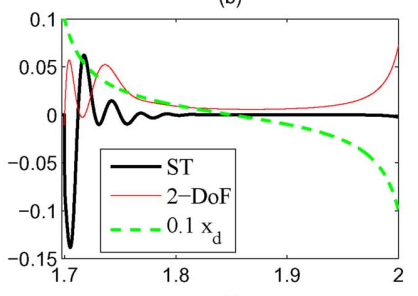

(d)

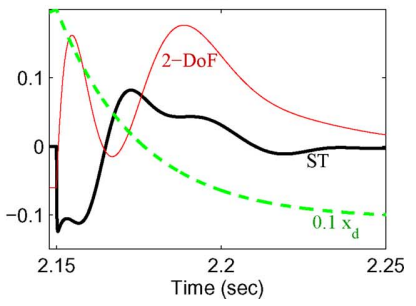

Fig. 15. Closeups of steady-state tracking errors in the absence of noise for robustified ST and ordinary 2-DoF systems, after a fivefold decrease in plant DC gain.

To investigate effects of disturbances in the robustified ST method, we applied step signals with amplitudes 10 and 1 at $1.1 \mathrm{~s}$ and $1.423 \mathrm{~s}$ to the input and output disturbance signals $d_{i}$ and $d_{o}$ in Fig. 9, respectively. The results, shown in Fig. 16, reveal effectiveness of the robustified ST method in rejection of the disturbances. The tracking errors marked "ST-1" in Fig. 17 show the initial decay of transients caused by the zero initial conditions of the plant at $t=0$ and by the input and output disturbances. To clarify the benefit of Filter $F(s)$ in the ST method, two other robustified ST designs; marked "ST-2" and "ST-3"; were considered with the filter replaced by a unity gain. With the same noise source, the projected noises in these ST methods have the same standard deviation as that of the previous ST method (ST-1). To obtain ST-2 from ST-1, we multiplied the PID controller gain by a factor of 1.3125 , and to obtain ST-3 we kept the PID controller of ST-1 intact and multiplied the double integral compensator by a factor of 1.3. The transients due to the foregoing ST methods (ST-2 and ST-3), which do not use the filter $F(s)$, as well as those of the ordinary

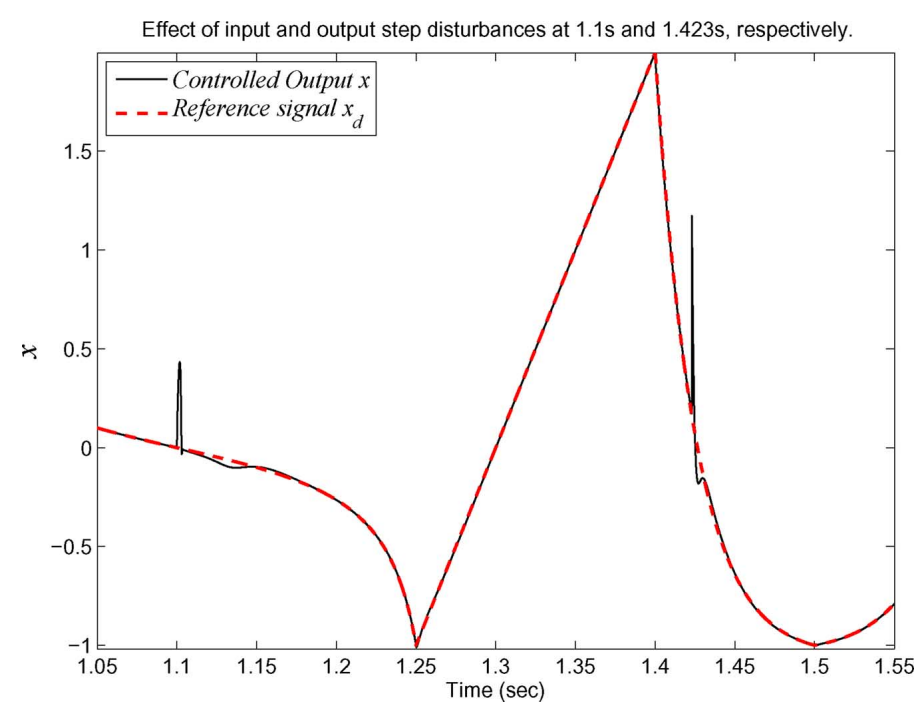

Fig. 16. Effect of input and output disturbance step signals on the controlled output in robustified ST system, applied with amplitudes 10 and 1 at $1.1 \mathrm{~s}$ and $1.423 \mathrm{~s}$, respectively.

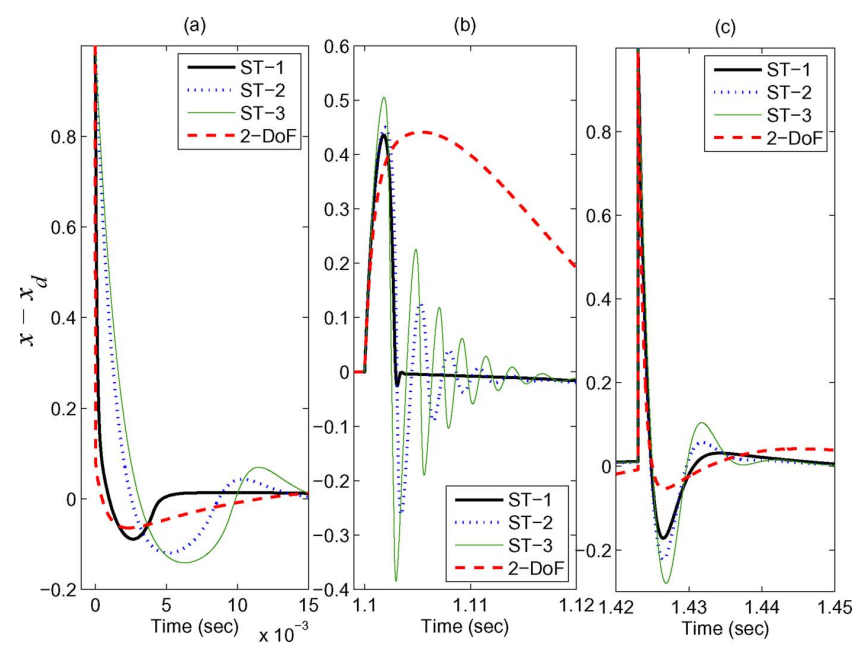

Fig. 17. Initial transients of the tracking error due to zero initial conditions of (a) the plant, (b) input disturbance, and (c) output disturbance.

2-DoF method, are also shown in Fig. 17. Clearly, appropriate use of filter $F(s)$ in the robustified ST method can considerably improve the transient performance of the ST system due to disturbances and initial conditions of the plant. Moreover, the input disturbance rejection performances for the ST methods are much better than that of the ordinary 2-DoF configuration, and so are their steady-state performances, as shown in Fig. 18(a).

To investigate the effect of unmodeled dynamics, we considered an additional pair of complex poles at $-700 \pm 6965 i$ for the plant while keeping its DC gain unity. Such unstructured uncertainty drives the previously designed ordinary 2-DoF system into instability while the robustified ST method (ST-1) still works in a satisfactory manner, as shown in Fig. 18(b), where square wave signals have also been applied as input and output disturbances. With the ST blocks replaced by unity gains in the ST system, the foregoing uncertainty does not noticeably change the phase margins but the gain margins of the inner and outer loops are reduced to $6.6 \mathrm{~dB}$ and $7.5 \mathrm{~dB}$, respectively. 
(a)
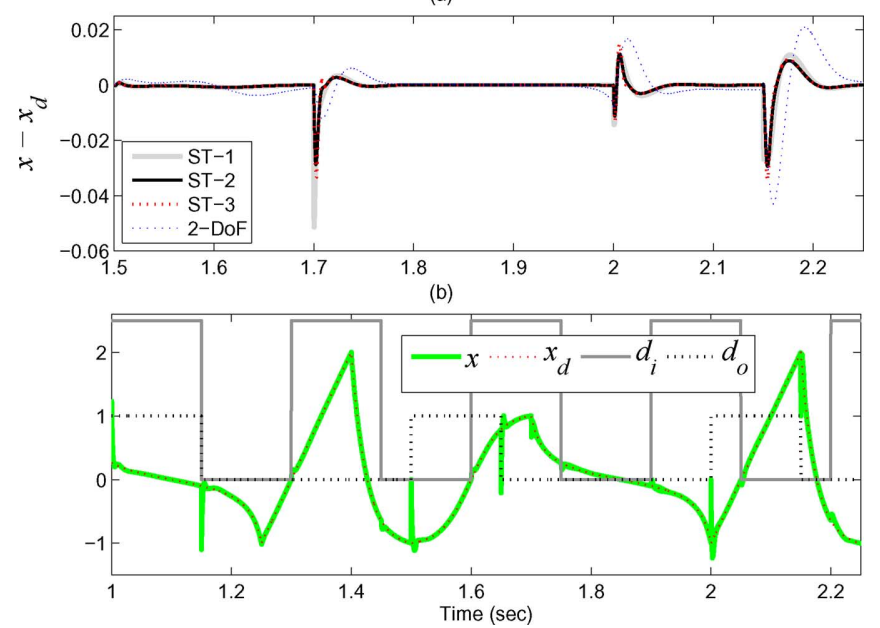

Fig. 18. (a) Time histories of steady-state errors for the three robustified ST systems, as well as the ordinary 2-DoF systems. (b) Tracking performance of the robustified ST method in the presence square-wave disturbances and unmodeled dynamics that render the ordinary 2-DoF system into instability.

\section{CONCLUSION}

Signal transformation (ST) method can improve the steadystate error in tracking of an arbitrary desired signal while limiting the closed-loop control bandwidth to prevent pollution of controlled output by the measurement noise. Sufficient conditions for the stability and convergence of the tracking error were obtained using singular perturbation theory. Effectiveness of the proposed method compared to ordinary 1-DoF and 2-DoF feedback systems, having similar projected noise levels, was examined by simulations. Using a stabilizing inner loop with integral action and an appropriately designed prefilter for reduction of noise level, the ST method can also provide acceptable robustness against uncertainties and disturbances. Although shown via simulations, a thorough mathematical analysis to address the superiority of the signal transformation (ST) method over ordinary feedback method is beyond the scope of the paper and is relegated to the future.

\section{APPENDIX}

In this section, a procedure is offered to obtain a state-space realization in the form of (13) for the plant, when the poles and zeros depend on a small variable $\varepsilon$ such that the plant transfer function approaches unity as $\varepsilon$ tends to zero. We assume that the plant has a strictly proper transfer function with unity DC-gain as

$$
G(s)=\frac{\prod_{k=1}^{m}\left(1+\frac{s}{z_{k}}\right)}{\prod_{k=1}^{n}\left(1+\frac{s}{p_{k}}\right)} .
$$

The poles and zeros of the plant depend on $\varepsilon$ in either of the following forms:

$$
\begin{gathered}
p_{k}=\left\{\begin{array}{l}
\frac{1}{\alpha_{k} \varepsilon} \\
\frac{1}{\alpha_{k} \varepsilon} \pm \omega_{k} i \\
\frac{1}{\alpha_{k} \varepsilon} \pm \frac{i}{\zeta_{k} \varepsilon}
\end{array}\right. \\
z_{k}=\left\{\begin{array}{l}
\frac{1}{\xi_{k} \varepsilon} \\
\frac{1}{\beta_{k} \varepsilon} \pm \mu_{k} i \\
\sigma_{k} \pm \frac{i}{\gamma_{k} \varepsilon}
\end{array}\right.
\end{gathered}
$$

where $\alpha_{k}, \omega_{k}, \zeta_{k}, \beta_{k}, \mu_{k}, \sigma_{k}$, and $\gamma_{k}$ are real numbers and $\xi_{k}$ is a real or complex number that are nonzero and do not depend on $\varepsilon$. The following block-upper-bidiagonal matrix is suggested as the state-matrix for the state-space realization:

$$
A_{p}=\left[\begin{array}{ccccc}
A_{1} & B_{1} & 0 & \ldots & 0 \\
0 & A_{2} & B_{2} & \ldots & 0 \\
0 & 0 & \ddots & \ddots & \vdots \\
0 & 0 & 0 & A_{l-1} & B_{l-1} \\
0 & 0 & 0 & 0 & A_{l}
\end{array}\right]
$$

where $l$ is number of poles when counting a pair of complexconjugate poles once. Each matrix $A_{k}(1 \leq k \leq l)$ is associated with a real pole or a complex pair. It is a $2 \times 2$ matrix for a complex pair and a scalar for a real pole such that its eigenvalues match with the associated poles. For repeated poles (real or complex), similar blocks are repeated equal to number of occurrence of the pole. Since the determinant of an upper-block-triangular matrix is equal to product of determinants of main diagonal blocks ([40, Eq. (2.7.6)]), the eigenvalues of the proposed state-matrix $A_{p}$ are equal to the plant poles. The elements of matrices $B_{k}$ are zero except the last element of their first column, which is equal to $1 / \varepsilon$. The following expressions describe matrix $A_{k}$ associated with the pole in (85):

$$
A_{k}=\frac{-1}{\alpha_{k} \varepsilon}, \text { or }\left[\begin{array}{cc}
\frac{-1}{\alpha_{k} \varepsilon} & \frac{\omega_{k}}{\varepsilon} \\
-\omega_{k} \varepsilon & \frac{-1}{\alpha_{k} \varepsilon}
\end{array}\right] \text {, or }\left[\begin{array}{cc}
\frac{-1}{\alpha_{k} \varepsilon} & \frac{1}{\zeta_{k} \varepsilon} \\
\frac{-1}{\zeta_{k} \varepsilon} & \frac{-1}{\alpha_{k} \varepsilon}
\end{array}\right] \text {. }
$$

The input matrix $B_{p}$ for the proposed realization is an $n \times 1$ vector as

$$
B_{p}=\left[\begin{array}{llll}
0 & \cdots & 0 & \frac{1}{\varepsilon}
\end{array}\right]^{T} .
$$

In this way, equalities $\widehat{A}_{p}(\varepsilon)=\varepsilon A_{p}(\varepsilon)$ and $\widehat{B}_{p}(\varepsilon)=\varepsilon B_{p}(\varepsilon)$ ensure that matrices $\widehat{A}_{p}(\varepsilon)$ and $\widehat{B}_{p}(\varepsilon)$ are sufficiently many times continuously differentiable functions of $\varepsilon \in\left[0, \varepsilon^{*}\right]$. The output matrix $\widehat{C}_{p}(\varepsilon)=C_{p}(\varepsilon)$ has $n$ elements to be determined from the numerator of the plant transfer function. The following example shows how the proposed $A_{p}(\varepsilon)$ and $B_{p}(\varepsilon)$ in (87) and (89) lead to a unique solution for $C_{p}(\varepsilon)$, whose elements are sufficiently many times continuously differentiable functions of $\varepsilon \in\left[0, \varepsilon^{*}\right]$. More rigorous proof for the foregoing assertion is still under investigation.

Example: We assume a ninth order plant having complex pair poles as $-1 / \varepsilon \pm \omega i$, repeated twice, another complex pair poles as $-1 / \alpha \varepsilon \pm i / \zeta \varepsilon$, two real poles at $-1 / \alpha_{2} \varepsilon$, and another real pole at $-1 / \alpha_{1} \varepsilon$, where $\alpha, \alpha_{1}, \alpha_{2}, \zeta$, and $\omega$ are nonzero constants. In this way, the integer $l$ is equal to 6 . We put the repeated complex pair at the very end of matrix $A_{p}$, then the other complex pair, repeated real poles, and finally the single real pole as the first element. In this way, submatrices $A_{k}, k \in$ $\{1, \ldots, 6\}$, and $B_{k}, k \in\{1, \ldots, 5\}$, are obtained as follows:

$$
\begin{aligned}
& A_{1}=\frac{-1}{\alpha_{1} \varepsilon}, B_{1}=B_{2}=\frac{1}{\varepsilon}, B_{3}=\left[\begin{array}{ll}
\frac{1}{\varepsilon} & 0
\end{array}\right] \\
& A_{2}=A_{3}=\frac{-1}{\alpha_{2} \varepsilon}, B_{4}=B_{5}=\left[\begin{array}{ll}
0 & 0 \\
\frac{1}{\varepsilon} & 0
\end{array}\right] \\
& A_{4}=\left[\begin{array}{ll}
\frac{-1}{\alpha \varepsilon} & \frac{1}{\zeta \varepsilon} \\
\frac{-1}{\zeta \varepsilon} & \frac{-1}{\alpha \varepsilon}
\end{array}\right], A_{5}=A_{6}=\left[\begin{array}{cc}
\frac{-1}{\varepsilon} & \frac{\omega}{\varepsilon} \\
-\omega \varepsilon & \frac{-1}{\varepsilon}
\end{array}\right] .
\end{aligned}
$$


The input matrix $B_{p}$ is selected in the form of (89) and the output matrix as

$$
C_{p}=\left[\begin{array}{llll}
c_{1} & c_{2} & \cdots & c_{9}
\end{array}\right]
$$

with $c_{k}, k \in\{1, \ldots, 9\}$, being unknown variables to be determined from plant's zeros. The numerator of the transfer function in terms of state-space matrices, when coefficient of the highest power of $s$ in denominator is unity, is equal to $C_{p}$ adj $(s I-$ $\left.A_{p}\right) B_{p}$, which can be written in the following form:

$$
\begin{aligned}
& \frac{c_{9}}{\varepsilon} s^{8}+\frac{\left(3+\frac{2}{\alpha}+\frac{2}{\alpha_{2}}+\frac{1}{\alpha_{1}}\right) c_{9}+\omega c_{8}}{\varepsilon^{2}} s^{7} \\
& +\left[\frac{\omega^{2} c_{9}}{\varepsilon}+\frac{f_{1}\left(\alpha, \alpha_{1}, \alpha_{2}, \zeta, \omega, c_{9}, c_{8}\right)+\omega c_{7}}{\varepsilon^{3}}\right] s^{6} \\
& +\left[\frac{f_{2}\left(\alpha, \alpha_{1}, \alpha_{2}, \omega, c_{9}, c_{8}\right)}{\varepsilon^{2}}\right. \\
& \left.+\frac{f_{3}\left(\alpha, \alpha_{1}, \alpha_{2}, \zeta, \omega, c_{9}, c_{8}, c_{7}\right)+\omega^{2} c_{6}}{\varepsilon^{4}}\right] s^{5} \\
& +\left[\frac{f_{4}\left(\alpha, \alpha_{1}, \alpha_{2}, \zeta, \omega, c_{9}, c_{8}\right)}{\varepsilon^{3}}\right. \\
& \left.+\frac{f_{5}\left(\alpha, \alpha_{1}, \alpha_{2}, \zeta, \omega, c_{9}, c_{8}, c_{7}, c_{6}\right)+\omega^{2} c_{5}}{\varepsilon^{5}}\right] s^{4} \\
& +\left[\frac{f_{6}\left(\alpha, \alpha_{1}, \alpha_{2}, \zeta, \omega, c_{9}, c_{8}\right)}{\varepsilon^{4}}\right. \\
& \left.+\frac{f_{7}\left(\alpha, \alpha_{1}, \alpha_{2}, \zeta, \omega, c_{9}, c_{8}, c_{7}, c_{6}, c_{5}\right)+\frac{\omega^{2}}{\zeta} c_{4}}{\varepsilon^{6}}\right] s^{3} \\
& +\left[\frac{f_{8}\left(\alpha, \alpha_{1}, \alpha_{2}, \zeta, \omega, c_{9}, c_{8}\right)}{\varepsilon^{5}}\right. \\
& \left.+\frac{f_{9}\left(\alpha, \alpha_{1}, \alpha_{2}, \zeta, \omega, c_{9}, c_{8}, c_{7}, c_{6}, c_{5}, c_{4}\right)+\frac{\omega^{2}}{\zeta} c_{3}}{\varepsilon^{7}}\right] s^{2} \\
& +\left[\frac{f_{10}\left(\alpha, \alpha_{1}, \alpha_{2}, \zeta, \omega, c_{9}, c_{8}\right)}{\varepsilon^{6}}\right. \\
& \left.+\frac{f_{11}\left(\alpha, \alpha_{1}, \alpha_{2}, \zeta, \omega, c_{9}, c_{8}, c_{7}, c_{6}, c_{5}, c_{4}, c_{3}\right)+\frac{\omega^{2}}{\zeta} c_{2}}{\varepsilon^{8}}\right] s \\
& +\frac{f_{13}\left(\alpha, \alpha_{1}, \alpha_{2}, \zeta, \omega, c_{9}, c_{8}, \ldots, c_{3}, c_{2}\right)+\frac{\omega^{2}}{\zeta} c_{1}}{\varepsilon^{9}} \\
& +\frac{f_{12}\left(\alpha, \alpha_{1}, \alpha_{2}, \zeta, \omega, c_{9}, c_{8}\right)}{\varepsilon^{7}}
\end{aligned}
$$

where functions $f_{1}, \ldots$, and $f_{13}$ linearly depend on the elements of $C_{p}$ and do not explicitly depend on $\varepsilon$. Having poles and zeros selected according to (85) and (86), respectively, and multiplying the numerator and denominator of (84) by $\prod_{k=1}^{n} p_{k}$ to make coefficient of the highest power of $s$ unity in the denominator, coefficient of $s^{l}$ in the numerator of plant transfer function can also be written in the following form:

$$
\frac{g_{l}(\varepsilon)}{\varepsilon^{n-l}}, l \in\{0,1, \ldots, m\}
$$

where $g_{l}(\varepsilon)$ is a well-defined function around $\varepsilon=0$ and sufficiently many times continuously differentiable. The coefficient defined in (92) should be equal to coefficient of $s^{l}$ in (91). Starting from the highest power of $s(l=n-1)$, the solution for unknown elements of $C_{p}$ are successively obtained. Since the highest powers of $\varepsilon$ in the denominators of (92) and coefficient of the unknown variable being solved ${ }^{4}$ are equal, the resulting solutions for elements of $C_{p}$ are well-defined functions around $\varepsilon=0$ and sufficiently many times continuously differentiable with respect to $\varepsilon$.

Remark: The procedure proposed here for realization of plant state-space equations in the form of (13) is also applicable if the plant is unstable and/or has a non-unity well-defined DC gain around $\varepsilon=0$.

\section{ACKNOWLEDGMENT}

The authors would like to thank E. Eleftheriou, H. Pozidis, and A. Sebastian for their support of this work.

\section{REFERENCES}

[1] S. Devasia, E. Eleftheriou, and S. O. R. Moheimani, "A survey of control issues in nanopositioning," IEEE Trans. Control Syst. Technol., vol. 15 , no. 5, pp. 802-823, Sep. 2007.

[2] Y. Sugimoto, P. Pou, O. Custance, P. Jelinek, M. Abe, R. Pérez, and S. Morita, "Complex patterning by vertical interchange atom manipulation using atomic force microscopy," Science, vol. 322, pp. 413-417, Oct. 2008.

[3] B. Bhushan, Nanotribology and Nanomechanics, An Introduction. New York: Springer-Verlag, 2005.

[4] S. Kalinin and A. Gruverman, Scanning Probe Microscopy: Electrical And Electromechanical Phenomena at the Nanoscale. New York: Springer, 2006.

[5] S. Salapaka and M. Salapaka, "Scanning probe microscopy," IEEE Control Syst. Mag., vol. 28, no. 2, pp. 65-83, Apr. 2008.

[6] S. Gonda, T. Kurosawa, and Y. Tanimura, "Mechanical performances of a symmetrical, monolithic three-dimensional fine-motion stage for nanometrology," Meas. Sci. Technol., vol. 10, pp. 986-993, 1999.

[7] J.-A. Kim, J. W. Kim, B. C. Park, and T. B. Eom, "Measurement of microscope calibration standards in nanometrology using a metrological atomic force microscope," Meas. Sci. Technol., vol. 17, no. 7, pp. 1792-1800, 2006.

[8] J. Kwon, Y.-S. Kim, K. Yoon, S.-M. Lee, and S. il Park, "Advanced nanoscale metrology of pole-tip recession with AFM," Ultramicroscopy, vol. 105, no. 1-4, pp. 51-56, 2005.

[9] F. Meli and R. Thalmann, "Long-range afm profiler used for accurate pitch measurements," Meas. Sci. Technol., vol. 9, no. 7, pp. 1087-1092, 1998.

[10] K.-B. Choi and J. J. Lee, "Passive compliant wafer stage for single-step nano-imprint lithography," Rev. Sci. Instrum., vol. 76, p. 075106, 2005.

[11] D. L. White and O. R. Wood, "Novel alignment system for imprint lithography," Rev. Sci. Instrum., vol. 18, no. 6, pp. 3552-3556, 2000.

[12] K. Miyahara, N. Nagashima, T. Ohmura, and S. Matsuoka, "Evaluation of mechanical properties in nanometer scale using AFM-based nanoindentation tester," Nanostruct. Mater., vol. 12, no. 5-8, pp. 1049-1052, 1999.

[13] I. Schmitz, M. Schreinera, G. Friedbachera, and M. Grasserbauer, "Phase imaging as an extension to tapping mode AFM for the identification of material properties on humidity-sensitive surfaces," Appl. Surface Sci., vol. 115, no. 2, pp. 190-198, 1997.

[14] A. Pantazi, A. Sebastian, G. Cherubini, M. Lantz, H. Pozidis, H. Rothuizen, and E. Eleftheriou, "Control of MEMS-based scanning-probe data-storage devices," IEEE Trans. Control Syst. Technol., vol. 15, no. 5, pp. 824-841, Sep. 2007.

[15] A. Pantazi, A. Sebastian, T. A. Antonakopoulos, P. Bächtold, A. R. Bonaccio, J. Bonan, G. Cherubini, M. Despont, R. A. DiPietro, U. Drechsler, U. Dürig, B. Gotsmann, W. Häberle, C. Hagleitner, J. L. Hedrick, D. Jubin, A. Knoll, M. A. Lantz, J. Pentarakis, H. Pozidis, R. C. Pratt, H. Rothuizen, R. Stutz, M. varsamou, D. Wiesmann, and E. Eleftheriou, "Probe-based ultrahigh-density storage technology," IBM J. Res. Dev., vol. 52, no. 4, pp. 493-511, 2008.

\footnotetext{
${ }^{4}$ Coefficient of $c_{l+1}$ in the coefficient of $s^{l}$ in (91).
} 
[16] A. Sebastian, A. Pantazi, S. O. R. Moheimani, H. Pozidis, and E. Eleftheriou, "Achieving subnanometer precision in a MEMS storage device during self-servo write process," IEEE Trans. Nanotechnol., vol. 7, no. 5, pp. 586-595, Sep. 2008.

[17] E. B. Brousseau, F. Krohs, S. Dimov, C. Griffiths, S. Scholz, A. Rees, and S. Fatikow, "Investigation of a new process chain based on atomic force microscopy scratching," in Proc. 4M/ICOMM '09-Global Conf. Micro Manuf., 2009, pp. 267-270.

[18] A. J. Fleming, A. Wills, and S. O. R. Moheimani, "Sensor fusion for improved control of piezoelectric tube scanners," IEEE Trans. Control Syst. Tech., vol. 16, no. 6, pp. 1265-1276, Nov. 2008

[19] O. J. M. Smith, Feedback Control Systems. New York: McGraw-Hill, 1958.

[20] N. C. Singer and W. P. Seering, "Preshaping command inputs to reduce system vibration," J. Dynamic Syst., Meas., Control, vol. 112, no. 1, pp. 76-82, 1990.

[21] J. Hyde and W. Seering, "Using input command pre-shaping to suppress multiple mode vibration," in Proc. IEEE Int. Conf. Robot. Autom., 1991, vol. 3, pp. 2604-2609.

[22] G. Mimmi and P. Pennacchi, "Pre-shaping motion input for a rotating flexible link," Int. J. Solids Structures, vol. 38, no. 10-13, pp. 2009-2023, 2001.

[23] E. Gilberta and I. Kolmanovskyb, "Nonlinear tracking control in the presence of state and control constraints: A generalized reference governor," Automatica, vol. 38, no. 12, pp. 2063-2073, 2002.

[24] P. Pennacchi, "Robustness of command input preshaping technique applied to residual vibration reduction," Shock Vibr., vol. 11, no. 3/4, pp. 377-382, 2004

[25] T. Sugie and H. Suzuki, "Robust reference shaping of periodic trajectories for systems with state/input constraints using impulse and step responses," in Proc. IEEE Conf. Decision Control, Dec. 2004, vol. 5, pp. 5511-5516.

[26] S. Rhim, S. G. Lee, and T. G. Lim, "Model-based reference trajectory generation for tip-based learning controller," J. Mech. Sci. Technol., vol. 19 , no. 1 , pp. 357-363, 2005.

[27] H. Suzuki and T. Sugie, "Off-line reference shaping of periodic trajectories for constrained systems with uncertainties," IEEE Trans. Autom. Control, vol. 53, no. 6, pp. 1531-1535, Jun. 2008.

[28] K. Kogiso and K. Hirata, "Reference governor for constrained systems with time-varying references," Robot. Auton. Syst., vol. 57, no. 3, pp. 289-295, 2009.

[29] C. Cao and A. Annaswamy, "A polynomial adaptive controller for nonlinearly parameterized systems," in Proc. IEEE Conf. Decision Control, 2006, pp. 1081-1086.

[30] D. S. Bayard, C.-H. C. Ih, and S. J. Wang, "Adaptive control for flexible space structures with measurement noise," in Proc. Amer. Control Conf., 1987.

[31] S. Tien, Q. Zou, and S. Devasia, "Iterative control of dynamics-coupling-caused errors in piezoscanners during high-speed AFM operation," IEEE Trans. Control Syst. Technol., vol. 13, no. 6, pp. 921-931, Nov. 2005.

[32] J. Yen, Y. Yeh, Y. Peng, and J. Lee, "Application of the continuous no-reset switching iterative learning control on a novel optical scanning system," Mechatronics, vol. 19, no. 1, pp. 65-75, 2009.

[33] G. Pipeleers, B. Demeulenaere, F. Al-Bender, J. De Schutter, and J. Swevers, "Optimal performance tradeoffs in repetitive control: Experimental validation on an active air bearing setup," IEEE Trans. Control Syst. Technol., vol. 17, no. 4, pp. 970-979, Jul. 2009.

[34] U. Aridogan, Y. Shan, and K. K. Leang, "Design and analysis of discrete-time repetitive control for scanning probe microscopes," $J$. $D y$ namic Syst., Meas., Control, vol. 131, no. 6, 2009.

[35] A. Sebastian and S. O. R. Moheimani, "Signal transformation approach to fast nanopositioning," Rev. Sci. Instrum., vol. 80, p. 076101, Jul. 2009.

[36] A. Bazaei, Y. K. Yong, S. O. R. Moheimani, and A. Sebastian, "Tracking control of a novel AFM scanner using signal transformation method," in Proc. 5th IFAC Symp. Mechatron. Syst., Cambridge, MA, Sep. 13-15, 2010.
[37] A. Bazaei, S. O. R. Moheimani, and A. Sebastian, "An analysis of signal transformation approach to triangular waveform tracking," $\mathrm{Au}$ tomatica, vol. 47, pp. 838-847, Apr. 2011.

[38] P. V. Kokotovic, J. O'Reilly, and H. K. Khalil, Singular Perturbation Methods in Control: Analysis and Design. Orlando, FL: Academic, 1986.

[39] H. Khalil, Nonlinear Systems. Englewood Cliffs, NJ: Prentice-Hall, 1996.

[40] D. S. Bernstein, Matrix Mathematics: Theory, Facts, and Formulas with Application to Linear Systems Theory. Princeton, NJ: Princeton Univ. Press, 2005.

[41] P. K. Sahoo and T. Riedel, Mean Value Theorems and Functional Equations. Singapore: World Scientific, 1998.

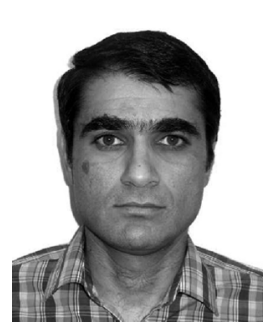

Ali Bazaei (M'10) received the B.Sc. and M.Sc. degrees in electrical engineering from Shiraz University, Shiraz, Iran, in 1992 and 1995, respectively, and $\mathrm{Ph}$.D. degrees in electrical engineering from Tarbia Modares University, Tehran, Iran, and the University of Western Ontario, London, ON, Canada, in 2004 and 2009, respectively.

From September 1995 to January 2000, he was an Instructor with Yazd University, Yazd, Iran. From September 2004 to December 2005, he was a Research Assistant in the Department of Electrical and Computer Engineering, University of Western Ontario. Since April 2009, he has been a Post-Doctoral Researcher with the School of Electrical Engineering and Computer Science, The University of Newcastle, Callaghan, Australia. His research interests lie in the general area of nonlinear systems including control and modeling of structurally flexible systems, friction modeling and compensation, and neural networks.

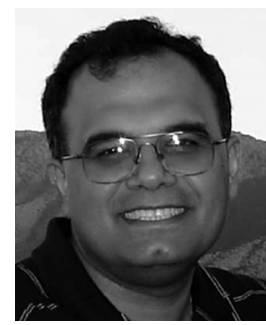

S. O. Reza Moheimani (M'97-SM'00-F'11) received the B.Sc. degree from Shiraz University, Shiraz, Iran, in 1990 and the M.Eng.Sc. and Ph.D. degrees from the University of New South Wales, Sydney, Australia, in 1993 and 1996, respectively, all in electrical and electronic engineering.

In 1996, he was a Postdoctoral Research Fellow at the School of Electrical and Electronic Engineering, Australian Defence Force Academy, Canberra, Australia. He joined The University of Newcastle, Callaghan, Australia, in 1997, where he founded and directs the Laboratory for Dynamics and Control of Nanosystems, a multi million dollar state of the art research facility dedicated to the advancement of nanotechnology through innovations in systems and control engineering. $\mathrm{He}$ is a Professor of electrical engineering and an Australian Research Council (ARC) Future Fellow. His current research interests are mainly in the area of dynamics and control at the nanometer scale, and include applications of control and estimation in nanopositioning systems for high-speed scanning probe microscopy, modeling and control of micro cantilever-based devices, control of electrostatic microactuators in microelectromechanical systems (MEMS), and control issues related to ultrahigh density probe-based data storage systems.

Prof. Moheimani is a Fellow of IFAC and a Fellow of the Institute of Physics (U.K.). He is a corecipient of the 2007 IEEE TRANSACTIONS ON CONTROL Systems TeChNOLOGY Outstanding Paper Award, and the 2009 IEEE CONTROL SYSTEMS TECHNOLOGY Award, the latter together with a group of researchers from IBM Zurich Research Labs, where he has held several visiting appointments. He has served on the editorial board of a number of journals including the IEEE TRANSACTIONS ON CONTROL SYSTEMS TECHNOLOGY, the IEEE/ASME TRANSACTIONS ON MECHATRONICS, and Control Engineering Practice, and has chaired several international conferences and workshops. 